\title{
Handelsbräuche bei Wagniskapitalfinanzierungen
}

\author{
Dr. Michael Denga, Berlin*
}

Inhaltsübersicht

ZGR 2021, 725-764

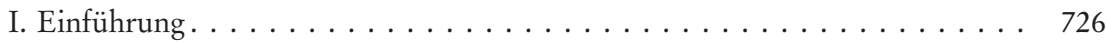

II. Unternehmensfinanzierung durch Wagniskapital . . . . . . . . . . . . . . . . 728

1. Geschäftsanteile als Finanzierungsinstrument . . . . . . . . . . . . . . 728

2. Geschäftsanteile als Instrument der Gründer- und Mitarbeiterbeteiligung . 731

III. Zentrales Beispiel: Vesting-Regime in der $\mathrm{GmbH}$. . . . . . . . . . . . . . . 734

1. Logik von Anteilsverlust und korrespondierenden Erwerbsrechten . . . . . 734

2. Ausgestaltung des Anteilsverlusts . . . . . . . . . . . . . . . . . . . . 736

3. Ausgestaltung der Entschädigung . . . . . . . . . . . . . . . 739

IV. Kautelarpraxis der Wagniskapitalfinanzierung . . . . . . . . . . . . . . 740

1. „Beteiligungs-Menüs“ aus Musterklauseln . . . . . . . . . . . . . . . . . . . . . . . 740

2. Einordnung als Handelsbrauch . . . . . . . . . . . . . . . . . 743

V. Wirtschaftssoziologischer Ansatz zur Hinauskündigungsrechtsprechung . . . 745

1. Offene Dogmatik des Gesellschafterausschlusses . . . . . . . . . . . . . . . . . 745

2. Alternative Wirksamkeitsmaßstäbe . . . . . . . . . . . . . . 747

3. Wirksamkeitsvermutung bei marktüblichen Klauseln . . . . . . . . . . . . 751

4. Neuralgische Regelungsthemen bei Wagniskapitalbeteiligungen . . . . . . . . 756

5. Umstandskontrolle . . . . . . . . . . . . . . . . . . . . . 759

6. Exemplarische Anwendung der modifizierten Hinauskündigungsdogmatik 760

VI. Fazit . . . . . . . . . . . . . . . . . . . . . . . . 763

Wagniskapitalinvestitionen fübren zu einer Funktionsänderung von Geschäftsanteilen. Bei den international verbreiteten Vesting-Regimen werden Geschäftsanteile insbesondere auch zu Finanzierungs- und Vergütungsinstrumenten. Die Verbreitung und Akzeptanz von diesbezüglichen Musterklauseln im Markt lässt auf Handelsbräuche schließen, die auch für die rechtliche Beurteilung der Beteiligungsregime maßgeblich sein müssen. Die für herkömmliche Ausschlussklausen entwickelte Hinauskündigungsrechtsprechung kann um ein wirtschaftssoziologisches Moment modifiziert werden, um die Interessen der Beteiligten besser abzubilden. Es sollte eine Wirksamkeitsvermutung bei marktüblichen Beteiligungsklauseln gelten und nicht mehr im Einzelfall nach einem sachlichen Grund für den Gesellschafterausschluss gesucht werden. Dieser wirtschaftsnahe Ansatz kann Rechtssicherheit steigern und das deutsche Gesellschaftsrecht im internationalen Wettbewerb stärken.

Venture capital investments lead to a change in the function of shares. In the case of the internationally widespread vesting regimes, shares in particular also become financing and remuneration

* Dr. iur., LL.M. (London), Maître en Droit (Paris), Wissenschaftlicher Mitarbeiter am Lehrstuhl von Prof. Dr. Dr. Stefan Grundmann, LL.M. (Berkeley), Humboldt-Universität zu Berlin. 
instruments. The dissemination and acceptance of such model clauses in the market suggests trade practices that must also be decisive for the legal assessment of the shareholding regimes. The case law on termination developed for conventional exclusion clauses can be modified to include a socio-economic element in order to better reflect the interests of the parties involved. A presumption of effectiveness should apply to participation clauses customary in the market and it should no longer be necessary to search for an objective reason for the exclusion of shareholders in individual cases. This business-oriented approach can increase legal certainty and strengthen German company law in international competition.

\section{Einfübrung}

Die Unternehmensfinanzierung durch „Wagniskapital“, besser bekannt als

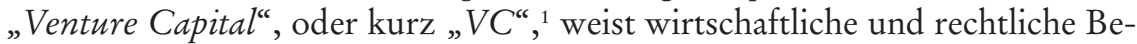
sonderheiten auf, die bislang nur vereinzelt aufgegriffen wurden - und bietet herausragendes Anschauungsmaterial sowie wesentliche Anstöße für den weiteren, immer noch ungelösten Fragenkreis zum „selbstgeschaffenen Recht der Wirtschaft".

Bei der Unternehmensfinanzierung durch Wagniskapital ist ein bedeutsamer Paradigmenwechsel zu beobachten. Gesellschaftsanteile werden insbesondere in jüngeren VC-finanzierten Unternehmen („Startups“) nicht allein wegen Gewinnausschüttungen oder der Mitwirkung am Gesellschaftsunternehmen erworben und eingeräumt. Sie übernehmen vielmehr die Funktion von Vergütungs- und Sicherungsmitteln. ${ }^{2}$ Die Gesellschafterstellung von Gründern und wichtigen Mitarbeitern wird dabei unter Berücksichtigung von Investoreninteressen als Investitionsordnung und antizipiertes Konfliktlösungsprogramm ausgestaltet.

Dabei spielt „Vesting“ eine besondere Rolle. Es handelt sich um ein kautelarjuristisch geprägtes Rechtsregime, welches durch im Markt verbreitete Musterklauseln umgesetzt wird. Sein abgestuftes Berechtigungs- und Disziplinierungsmodell mit einer Logik des "Erdienens von Anteilen“ verspricht große Gewinne im unternehmerischen Erfolgsfall. Es kann allerdings auch zum voll-

1 Zur Entstehung von Venture Capital aus wirtschaftssoziologischer Perspektive siehe bei KüHL, Exit, 2003, S. 27; aus juristischer Perspektive zur Begriffseingrenzung des Venture Capital, vgl. KunTZ, Gestaltung von Kapitalgesellschaften zwischen Freiheit und Zwang, 2016, Einleitung, A. II.; Weitnauer, in: Weitnauer, Handbuch Venture Capital, 6. Aufl., 2019, Rdn. $1 \mathrm{ff}$; schon früh: SCHÄFER/STEPHAN, Venture-Capital-Verträge, 2003, S. 1 ff; aus ökonomischer Sicht umfassend der Band von Cumming (Hrsg.), The Oxford Handbook of Venture Capital, 2012; die fehlende Untersuchung auch im US-Recht beklagend, Bengtsson, in: Cumming, aaO, S. $478 \mathrm{ff}$; mit dem Praxisbeispiel des Silicon Valley, FerRary/GranovetTer, Economy and Society, 2009, vol. 38, no. 2, 326, $341 \mathrm{ff}$.

2 Siehe etwa KüHL, aaO (Fn. 1), S. 19. 
ständigen Verlust aller Unternehmensanteile führen. Vesting wirft daher vor dem Hintergrund der wechselhaften Rechtsprechung zum Gesellschafterausschluss und der damit zusammenhängenden Abfindungsproblematik eine Vielzahl von Rechtsfragen auf. Es soll hier wegen seiner weiten Verbreitung und noch ungeklärten höchstrichterlichen Bewertung beispielhaft auch für andere, in VC-Beteiligungsklauseln verbreitete, Kautelarlösungen analysiert werden.

Im Zentrum steht dabei die Legitimationsfrage privat konstituierter Regeln genauer: der Mehrheitsmacht, einen einzelnen Gesellschafter auszuschließen. ${ }^{3}$ Da VC-Beteiligungen Paradebeispiele handelsgeschaffenen Privatrechts sind, bieten sie neuerliche Gelegenheit zur Analyse der Funktions- und Wirkbedingungen von Selbstregulierung im Unternehmensrecht. ${ }^{4}$ Besonders bedeutsam ist dabei, dass die Parteien von VC-Finanzierungen sehr bewusst Risiken eingehen und als professionell zu charakterisieren sind; statt strukturellem Machtungleichgewicht, wie zwischen Unternehmern und Verbrauchern, herrscht zwischen Investoren, Gründern und zentralen Mitarbeitern weitestgehend Waffengleichheit. "Selbstgeschaffenes Recht der Wirtschaft ${ }^{\text {“5 }}$ wird in seiner Manifestation als Handelsbrauch bislang vor allem lediglich als Mittel der Auslegung von Rechtsgeschäften und der Pflichtkonkretisierung anerkannt.

Hier wird eine weitergehende These aufgestellt, die dem professionellen Kontext der Investitionsprogramme besser gerecht werden soll: die Umsetzung von VC-Beteiligungen durch marktübliche Musterklauseln begründet eine Wirksamkeitsvermutung. Bei der Kontrolle am Maßstab gesetzlicher Generalklauseln ist auf diese Weise das Faktum des normativ begründeten Handelsbrauchs unter professionellen Parteien angemessen abgebildet. Insbesondere bei der Hinauskündigungsrechtsprechung, ausgehend von ihrem dogmatischen Sockel, der Sittenwidrigkeit, reduzieren die anzutreffenden Handelsbräuche die Prüfungsdichte. Denn in den VC-Vertragsmustern inkorporierte Verkehrssitten verstoßen nicht gegen die guten Sitten - sondern konkretisieren sie. Bezogen auf das für die Sittenwidrigkeitskontrolle maßgebliche Instrument der Interessenabwägung bedeutet dies die Vermutung eines Interessengleichgewichts.

Andererseits kann die marktunübliche Verwendung von Musterklauseln im Einzelfall zu ihrer Sittenwidrigkeit führen. In solchen Fällen wäre die Vermutung eines Interessengleichgewichts widerlegt. Um in dieser Hinsicht rechtssichere Gestaltungen zu fördern, regt der Beitrag eine Systematisierung typischerweise kritischer Umstände bei VC-Beteiligungen an. Im Vordergrund

3 Zum binären Legitimierungsmodell nach Zustimmung und Gemeinwohlbelangen, BACHmann, Private Ordnung, 2006, insbes. S. 68, $363 \mathrm{ff}, 393 \mathrm{ff}$.

4 Vgl. dazu Merkт, in: Bumke/Röthel, Autonomie im Recht, 2017, S. 167, 169.

5 Dazu früh schon Großmann-Doerth, Selbstgeschaffenes Recht der Wirtschaft und staatliches Recht, 1933 und RüHL, Rechtsschöpfung durch die Wirtschaft, 1931. 
rechtlicher Auseinandersetzungen würde insgesamt weniger die individuelle Interessenlage als vielmehr die Marktüblichkeit der betreffenden Klausel stehen. Durch diesen Ansatz könnten sich Gerichte stärker der Wirtschaftspraxis zuwenden - nicht zuletzt auch, um auf längere Sicht die Dominanz der Schiedsgerichtsbarkeit bei VC-Finanzierungen $\mathrm{zu}$ brechen und deutsches Recht für internationale Wagniskapitalinvestitionen attraktiv zu halten.

Zunächst ist die Gemengelage wirtschaftlicher Interessen und Verkehrssitten bei VC-Finanzierungen nachzuzeichnen, die zu erheblichen Verschiebungen im Gebrauch gesellschaftsrechtlicher Instrumente führt (II.). Hiernach sind, stellvertretend für andere Beteiligungsinstrumente des Wagniskapitals, Logik und Struktur von Vesting-Regimen als empirischer Befund darzustellen (III.). Zentral ist die Analyse der VC-spezifischen Kautelarpraxis, welche den wirtschaftlichen Interessenausgleich in Handelsbräuche kondensiert (IV.) Aus dieser Erkenntnis heraus kann ein wirtschaftssensibler Maßstab für die Wirksamkeitskontrolle von Ausschlussklauseln gewonnen und exemplarisch durchgespielt werden (V.).

\section{Unternehmensfinanzierung durch Wagniskapital}

Die Unternehmensfinanzierung durch Wagniskapital hat zu einem bedeutsamen Paradigmenwechsel geführt - Geschäftsanteile werden nicht mehr allein als Verkörperung der gesellschaftsrechtlichen Mitgliedschaft eingesetzt, sondern als marktbezogenes Finanzierungs- und Vergütungsinstrument.

\section{Geschäftsanteile als Finanzierungsinstrument}

VC-Finanzierungen haben inzwischen eine bedeutende Rolle für Gründung und Wachstum von Startups. Für das allgemeine Problem der Investitionssicherung lautet die VC-spezifische Lösungsformel verkürzt „Finanzierung gegen Gesellschaftsbeteiligung“ statt wie hergebracht „Kredit gegen Zins und Sicherung".

\section{a) Markt für Wagniskapital}

Für Unternehmensgründer ist es häufig schwer herkömmliche Bankdarlehen zu erhalten, wenn ihr Unternehmen sich auf neuartige Produkte stützt oder in Märkten mit starkem Wettbewerb bestehen soll. ${ }^{6}$ Der wirtschaftliche Erfolg

$6 \mathrm{Zu}$ den verschiedenen Phasen der Gründungsfinanzierung vgl. Möllmann/Möllmann, BWNotZ 2013, 74, $77 \mathrm{ff}$. 
technischer oder betriebswirtschaftlicher Innovation ist meist ungewiss. ${ }^{7}$ In kompetitiven Märkten ist daneben unsicher, ob noch Raum für Mitbewerber ist oder bereits ein „Platzhirsch“ wegen Netzwerkeffekten ${ }^{8}$ unaufhaltbar auf eine Monopolstellung zusteuert. ${ }^{9}$ In diesen Fällen stehen hoher Kapitalbedarf und hohes Ausfallrisiko nebeneinander. ${ }^{10}$

Banken können dabei auf Grundlage herkömmlicher Darlehensmodelle Kredit weder konform mit den Kreditvergabeleitlinien der Europäischen Bankenaufsichtsbehörde, geschweige denn in profitabler Weise vergeben, da sie anders als bei etablierten Unternehmen meist auch nicht auf betriebliche Sicherungsmittel, wie Grundstücke oder Kundenforderungen, zugreifen können. ${ }^{11}$ Die Risiken ließen sich nur durch wuchernahe Zinsen kompensieren. Dieser Mangel an Finanzierungsmöglichkeiten wird nur teilweise durch die öffentliche „Förderlandschaft" aufgefangen. ${ }^{12}$ Der drängende Finanzierungsbedarf auf Gründerseite, freilich auch das Erfolgspotential neuer Unternehmungen, hat bereits Mitte des 20. Jahrhunderts zur Herausbildung von auf Risikobranchen spezialisierter Wagniskapitalgeber geführt, welche das hier zentrale Modell „Finanzierung gegen Gesellschaftsbeteiligung" weitestgehend ohne staatlichen Einfluss geprägt haben. ${ }^{13}$ Zudem sind Wagnisinvestitionen aus Perspektive etablierter Unternehmen eine Möglichkeit für outgesourcte Innovationsarbeit, weshalb auch sie relevante Akteure sind. ${ }^{14}$

7 Vgl. etwa bei Bandulet, Finanzierung technologieorientierter Unternehmensgründungen, 2005, S. $7 \mathrm{ff}, 56 \mathrm{ff}$.

$8 \mathrm{Zu}$ diesen, vgl. Ewald, in: Wiedemann, Handbuch des Kartellrechts, 4. Aufl., 2020, $\$ 7$ Rdn. $68 \mathrm{ff}$; zur ökonomischen Theorie THum, Netzwerkeffekte, Standardisierung und staatlicher Regulierungsbedarf, 1995; zur inflationären Berufung auf diese durch Unternehmen, The Economist, 20. April 2019, S. 23 ff; kritisch auch KüHL, aaO (Fn. 1), S. $103 \mathrm{ff}$.

9 Vgl. KÜHL, aaO (Fn. 1), S. 14 ff; vgl. m. w. N. auch DengA, ZGE 12 (2020), 12 f.

10 Brettel/Rudolf/Witt, Finanzierung von Wachstumsunternehmen, 2005, S. $13 \mathrm{ff}$.

$11 \mathrm{Zu}$ den aktuellen Kreditvergaberichtlinien der EBA vgl. den Final Report, Guidelines on loan origination and monitoring, Mai 2020, EBA/GL/2020/06; siehe auch GroH, in: Cumming, The Oxford Handbook of Venture Capital, 2012, S. 15 ff; vgl. Möllmann/ Möllmann, BWNotZ 2013, 74, 77 ff; Schäfer/Stephan, aaO (Fn. 1), S. 2.

12 Vgl. dazu Brettel/Rudolf/WitT, aaO (Fn. 10), S. 179 ff; zu Risikokapitalbeihilfen, vgl. Zuleger/Wendland, in: von der Groeben/Schwarze/Hatje, Europäisches Unionsrecht, 7. Aufl., 2015, Art. 107 Rdn. $499 \mathrm{ff}$.

13 Vgl. dazu Kühl, aaO (Fn. 1), S. 14 ff; siehe auch bei Weitnauer, aaO (Fn. 1), Rdn. 29 ff; zur deutschen Praxis siehe insbesondere Antonczyk/Breuer/Mark, FB 2008, 225, 230; SchefсZYк, Erfolgsstrategien deutscher Venture Capital-Gesellschaften, 3. Aufl., 2004, S. $61 \mathrm{ff}$.

14 Vgl. etwa die Selbstbeschreibung des Pharmakonzerns Merck, abrufbar unter: https:// www.merckgroup.com/de/company/partnering/m-ventures.html (letzter Zugriff: 4.1. 2021). Die Investition von etablierten Unternehmen wird als Corporate-VC bezeichnet und erfolgt meist im Verbund mit der Investition durch VC-Fonds; dazu BessLeR/HoL- 
VC ist im gegenwärtigen Niedrigzinsumfeld von besonders hohem Renditedruck getrieben ${ }^{15}$ - auf VC-Investitionen spezialisierte Fonds versprechen ihren Geldgebern dennoch erhebliche Vermögenszuwächse durch Investitionen in Startups. Dabei haben sich deren Bewertungsmaßstäbe von Profitabilität auf Wachstum verlagert, weshalb „blitzscaling“ von Unternehmen, insbesondere von sog. "unicorns“, zu beobachten ist. ${ }^{16} \mathrm{Ob}$ dies "nachbaltigem Wirtschaften “ entspricht, wie es zunehmend gesetzgeberische Intention wird, ${ }^{17}$ scheint fraglich. Bei der Kapitalvergabe müssen Wagniskapitalgeber jedenfalls Verluste und sogar Total-Ausfälle in einem Großteil ihrer Investitionen einkalkulieren. Sie sind daher auf Verlustkompensation durch einzelne besonders erfolgreiche Projekte angewiesen. ${ }^{18}$

\section{b) Sicherungsinteresse der Investoren}

Den großen Finanzierungsrisiken begegnen VC-Investoren damit, Unternehmensanteile als Sicherheits-Ersatz im Gegenzug für eine Start- oder Zwischenfinanzierung anzunehmen. Das Engagement der Wagniskapitalgeber ist dabei von vornherein auf einen „Exit" angelegt - die Beteiligung soll gewinnbringend verkauft werden, im Idealfall anlässlich eines Börsengangs. ${ }^{19} \mathrm{Die}$ Wagniskapitalgeber unterstützen die finanzierten Unternehmen auch fachlich und durch Branchenvernetzung, um deren Erfolg sicherzustellen und Wettbewerbsdefizite gegenüber etablierten Unternehmen zu kompensieren. ${ }^{20} \mathrm{Zur}$

Ler/Seim/Zimmermann, in: Cumming, The Oxford Handbook of Venture Capital, 2012, S. $627 \mathrm{ff}$.

15 Vgl. BMF, Herausforderungen der Niedrigzinsphase für die Finanzpolitik, 2/2017, S. 9 ff, abrufbar unter: https://www.bundesfinanzministerium.de/Content/DE/Downloads/ Broschueren_Bestellservice/2017-06-28-wissenschaftlicher-beirat-niedrigzinsphase.pdf? blob=publicationFile Ev $=5$ (letzter Zugriff: 4.1.2021).

16 Vgl. etwa Kenney/Zysman, Unicorns, Cheshire Cats and the New Dilemmas for Entrepreneurial Finance?, BRIE Working Paper 2018/1; kritisch mit einer umfassenden Analyse KüHL, aaO (Fn. 1); aktuell: The Economist, 20. April 2019, S. 11, 23 ff. Zu den ökonomischen Grundlagen der für Stakeholder zentralen Unternehmensreputation, vgl. KLÖHN/Schmolke, NZG 2015, 689, insbes. $691 \mathrm{f}$.

17 Siehe etwa den Aktionsplan: Finanzierung nachhaltigen Wachstums der EUROpäISCHEN Kommission, COM(2018) 97 final; dazu Möslein/MitTwoch, WM 2019, 481 und schon früh Steinmann, Das Großunternehmen im Interessenkonflikt, 1969; m. w. N. WiedeMANN, ZGR 1980, 147, $161 \mathrm{ff}$.

18 KüHL, aaO (Fn. 1), S. 15 ff.

19 Vgl. etwa Schäfer/Stephan, aaO (Fn. 1), S. 2. Auch dies stellt die Nachhaltigkeit des Kapitaleinsatzes in Frage, kritisch dazu KüHL, aaO (Fn. 1), S. 17 ff; grundsätzlich ist die Tätigkeit der Wagniskapitalgeber durch die AIFM-Richtlinie 2011/61/EU erfasst.

20 Zum "Postinvestment Involvement" vgl. CHuA/Wu, in: Cumming, The Oxford Handbook of Venture Capital, 2012, S. $722 \mathrm{ff}$. 
Investitionssicherung schaffen die Wagniskapitalgeber Kontrollstrukturen, die sich auf gemeinsame Geschäftspläne beziehen oder eine umfassende Berichterstattung und ständigen persönlichen Kontakt mit der Geschäftsführung beinhalten. Finanzierungstranchen können von „Mile-Stones“ im Geschäftsplan und Reportpflichten abhängig gemacht werden. ${ }^{21}$ In Ausnahmefällen können sogar Vetorechte der Kapitalgeber gegenüber der Geschäftsführung vereinbart werden. Mit ihren Stimmrechten in der Gesellschaft und ihrer ggf. faktischen Macht in Hinblick auf anstehende Folgefinanzierungen (samt des Phänomens „vorauseilenden Gehorsams“) können sie auch die Entlassung der Geschäftsführer herbeiführen. Dies ist aus Investorenperspektive wesentlich, denn der Unternehmenserfolg hängt in der Gründungsphase von den Persönlichkeiten der Gründer und zentralen Mitarbeiter ab; diese sind souveräne und verhandlungsmächtige Zentralgestalten im Startup. ${ }^{22}$ Darin liegt ein wesentlicher Unterschied zu etablierten Unternehmen, bei denen eine Organisation besteht, die auch bei mehrfacher Auswechslung von Spitzen und Fachpersonal erfolgreich operieren kann. Bei Startup-Investitionen ist daher aus Investorensicht wünschenswert, die Gründer und zentralen Mitarbeiter an das Unternehmen persönlich zu binden - sowie fundamentale Meinungsverschiedenheiten effektiv auszuschließen..$^{23}$ Der betroffene Gesellschafter hingegen hat ein wirtschaftliches Interesse am Fortbestand seiner Beteiligung und an einer Verfahrenshoheit im Ausschlussstreit. ${ }^{24}$ Dieser Interessenkonflikt schlägt sich in der typischen Ausgestaltung von Wagniskapitalbeteiligungen nieder. ${ }^{25}$

\section{Geschäftsanteile als Instrument der Gründer- und Mitarbeiterbeteiligung}

Auch gegenüber geschäftsführenden Gründern und Mitarbeitern fördern die Schwierigkeiten junger Unternehmen bei der Kapitalbeschaffung den unkonventionellen Einsatz von Geschäftsanteilen. Dabei wird das Modell „Arbeit ge-

21 Dazu etwa Hellmann/Puri, The Journal of Finance, Volume 57, Issue 1, Feb. 2002, $169 \mathrm{ff}$.

22 KüHL, aaO (Fn. 1), S. 46 ff, 67 ff.

23 Dieses Interesse erkennt auch der BGH als legitim an, vgl. BGH NZG 2005, 968, 970; vgl. dazu auch Bank/Möllmann, Venture Capital Agreements in Germany, 2017, Teil 3 Rdn. 239ff; STEnZel, in: Holzner/Mantke/Stenzel, Handbuch Managementbeteiligungen, 2017, Rdn. 609; SAmios/Arnold, Dealterms.vc, 2019, S. 145 ff, 152; vgl. kritisch, mit weiteren Nachweisen zur Legitimität des Funktionsinteresses als Ausschlussmotiv, Grunewald, Der Ausschluß aus Gesellschaft und Verein, 1987, S. $17 \mathrm{ff}$.

24 Vgl. Kästle/Heuterkes, NZG 2005, 289.

25 Siehe auch Wenzel, in: Drygala/Wächter, Venture Capital, Beteiligungsverträge und „Unterkomplexitätsprobleme“, 2018, S. 77 ff, der die Perspektive eines Interessengleichlaufs zwischen Investoren und Gründern vertritt. 
gen Gesellschaftsbeteiligung" praktiziert. ${ }^{26}$ Häufig können Startups weder kompetitive Gehälter zahlen noch vergleichbare Sicherheit oder Karriereperspektiven bieten wie etablierte Unternehmen. Gerade zu Beginn der Geschäftstätigkeit müssen die verfügbaren Ressourcen oftmals vollständig auf die operative Geschäftsentwicklung konzentriert werden; geschäftsführende Gründer und Mitarbeiter verzichten in dieser Phase häufig auf ein marktübliches Gehalt. ${ }^{27}$ Die erfolgsbestimmende längerfristige Motivation und Loyalität können in dieser Situation über eine Beteiligung am Unternehmen hergestellt werden. ${ }^{28}$ Entsprechende Mitarbeiterbeteiligungsprogramme treten in verschiedenen Formen auf und beschäftigen neuerdings auch das BMWi sowie das BMF. ${ }^{29}$

Verbreitet sind sog. „virtuelle Beteiligungen“ als schuldrechtlicher Differenzzahlungsanspruch bezogen auf die Entwicklung eines fiktiven Anteilswertes, daneben auch Anwartschaften auf Anteile, bedingte Anteilsübertragungen wie beim sogleich darzustellenden Vesting und seltener auch bedingungslose Beteiligungen. ${ }^{30}$ Der besondere Anreiz für Gründer und Mitarbeiter ergibt sich in allen Modellen aus der Aussicht auf Beteiligung an einer durch sie selbst beeinflussbaren positiven Unternehmensentwicklung. Das primäre ökonomische Interesse der Gesellschafter liegt nicht in der Gewinnausschüttung, sondern im Weiterverkauf der Beteiligung, da eine Steigerung des Anteilswerts innerhalb der Vesting-Periode erhofft wird. Mit dieser utilitaristischen Inzentivierung kann

26 Vgl. etwa KüHL, aaO (Fn. 1), S. 174f; Rödding/Brinkmann/Fischer, in: Lüdicke/Sistermann, Unternehmenssteuerrecht, 2. Aufl., 2018, $\mathbb{1 4}$ Rdn. 145; Schniepp/Giesecke, NZG 2017, 128.

27 In etablierten Unternehmen dienen Mitarbeiter- und Managementbeteiligungen der Vergütungsergänzung durch Gewinnausschüttung, sowie der politischen Stärkung des Beteiligten nach innen und außen; vgl. dazu BGH NJW 2005, $3641 \mathrm{f}$ - Mediamarkt-Saturn; Habersack/Verse, ZGR 2005, 451, 472ff; Weitnauer/Grob, GWR 2015, 353, 354; Schockenhoff, NZG 2018, 201, 207; Stenzel, aaO (Fn. 23), Rdn. $638 \mathrm{ff.}$

28 Vgl. zu den Motivationswirkungen von Mitarbeiterbeteiligungen auch HABERSACK/VERSe, ZGR 2005, 451, 455; Weitnauer, aaO (Fn. 1), Teil F Rdn. 220.

29 Siehe dazu Lowitzsch, Studie im Auftrag des BMWi, Verbreitung der Mitarbeiterkapitalbeteiligung in Deutschland und Europa, 2020; Referentenentwurf des BMF „zur Stärkung des Fondsstandorts Deutschland“, Dezember 2020, dort insbesondere $₫ 19$ a EStG$\mathrm{E}$ (inzwischen umgesetzt durch das Fondsstandortgesetz vom 3. Juni 2021); siehe auch die Studie \#ESOPasap vom Bundesverband Deutsche Startups e.V., 2020; RödDing/ Brinkmann/Fischer, aaO (Fn. 26), $\mathbb{1} 14$ Rdn. 139ff; Stenzel, DStR 2018, 82 und 139; SchanZ, NZA 2000, 626; vgl. zu virtuellen Anteilen, Schniepp/Giesecke, NZG 2017, 128; besonders etabliert sind Managementbeteiligungen, vgl. unter ausdrücklicher Bezugnahme auf Vesting-Programme Europäische Kommission, DG JUST/A.3, Guidelines on the standardised presentation of the remuneration report under Directive 2007/ 36/EC, p. $9 \mathrm{ff}$.

30 Vgl. den Überblick bei Rödding/Brinkmann/Fischer, aaO (Fn. 26), $\mathbb{1} 14$ Rdn. $139 \mathrm{ff}$; umfassend auch Holzner/Mantke/Stenzel, Handbuch Management-Beteiligungen, 2017, mit einem Überblick in Teil A, S. 1-12. 
das alte Principal-Agent-Dilemma im neuen Kontext gelöst und die Interessen von Gründern und Investoren am Unternehmenserfolg gleichgerichtet werden („Alignment of Interest“). Die Steigerung des Anteilswerts zum geteilten Nutzen von Gründern, wesentlichen Mitarbeitern und Investoren ist dabei zentral. ${ }^{31}$

Durch die Modelle der Mitarbeiterbeteiligung, deren ökonomische Rationale auch auf finanzierungsabhängige Gründer zutrifft, wird bisweilen sogar behauptet, die Trennung zwischen Angestellten und Gesellschaftern sei aufgehoben und es handele sich um "Unternehmer im Unternehmen“. ${ }^{32}$ Verbunden mit dem Risiko unternehmerischen Misserfolges in kompetitiven Märkten, ergibt sich jedenfalls ein für alle Beteiligten klares spekulatives Moment, ${ }^{33}$ was auch für die rechtliche Behandlung von VC-Beteiligungsprogrammen von Bedeutung sein muss.

Hier sollen Vesting-Programme in der $\mathrm{GmbH}^{34}$ im Vordergrund stehen. Sie bewirken tiefgreifende Modifikationen am herkömmlichen Verständnis des Gesellschafterstatus, die bislang in der Rechtswissenschaft noch zu wenig beachtet wurden und in der Praxis häufig Probleme bereiten. Vesting-Anteile stellen ein Minus zur bedingungslosen Gesellschaftsbeteiligung dar. Der gesellschaftsrechtliche Status als dinglicher Anteil ist dabei nicht erodiert, wie es etwa bei bloßen Optionen, virtuellen Anteilen, Beteiligungen an Mitarbeiterbeteiligungsvehikeln - und seltenen - stillen Beteiligungen der Fall ist. ${ }^{35}$ Sie

31 So auch Kästle/Heuterkes, NZG 2005, 289; Wenzel, aaO (Fn. 25), S. 77 ff; zum Principal-Agent-Konflikt, siehe Jensen/Meckling, Journal of Financial Economics 3 (1976), 305, 308 ff; Kraakman u.a., The Anatomy of Corporate Law, 3rd ed. 2017, Chapter 2; WindBichleR, Gesellschaftsrecht, 24. Aufl., 2017, $\$ 1$ Rdn. 30.

32 KüHL, aaO (Fn. 1), S. 132 ff.

33 Vgl. KüHL, aaO (Fn. 1), S. 52 ff; Janeway, Doing Capitalism in the Innovation Economy, 2. Aufl., 2018, S. 153 ff; zu den Mechanismen der Spekulation und deren Bedeutung für Marktinformationspflichten, KLöHN, Kapitalmarkt, Spekulation und Behavioral Finance, 2006, $\mathbb{S}$ 4. Zu den Problemen der Unternehmensbewertung Wollny, in: Drygala/ Wächter, Venture Capital, Beteiligungsverträge und „Unterkomplexitätsprobleme“, 2018, S. 3 ff; Schäfer/Stephan, aaO (Fn. 1), S. 19 ff; Gehrlein, WM 2019, 1, 8 f.

34 Die $\mathrm{GmbH}$, mit ihrer Unterform der UG, ist die bei VC-Finanzierungen am häufigsten gewählte Gesellschaftsform; vgl. die Studie von PricewaterhouseCoopers, Startupunternehmen in Deutschland, 2017, S. 32; Mailänder, in: in: Weitnauer, Handbuch Venture Capital, 6. Aufl., 2019, Teil E Rdn. 223 ff; mit starkem Praxisbezug, SchöNHAAR, VC-Magazin, Sonderausgabe Oktober 2014; siehe freilich KunTZ, aaO (Fn. 1), der die im US-Recht vor einigen Jahren verbreitete Umsetzung von VC-Investitionen in Public Companies bzw. Limited Liability Companies abbildet; siehe zur Entwicklung der gesellschaftsrechtlichen Rahmenbedingungen, insbesondere zur „kleinen Aktiengesellschaft“ Weitnauer, aaO (Fn. 1), Rdn. 91 ff; den Bedeutungsverlust der AG in Folge der Dotcom-Blase antizipierend, Schäfer/Stephan, aaO (Fn. 1), S. 16 f.

35 Siehe dazu insbesondere das Praktikerhandbuch Holzner/Mantke/Stenzel, aaO (Fn. 30); umfassend zur Problematik der Eigentümerstellung im Unternehmen, vgl. Hansmann, Journal of Law, Economics, and Organization vol. 4, no. 2 Fall 1988, S. 267. 
sind dinglich vollwertige Anteile, die bei Eintritt bestimmter Ereignisse an die Gesellschaft oder Dritte fallen können. Beim Einstieg von Investoren in Startups sowie als Vergütungsmodell für Gründer und Mitarbeiter ist Vesting ein zentrales und fest etabliertes Element der gesellschaftsrechtlichen Kautelarpraxis. ${ }^{36}$ Vesting soll hier pars pro toto behandelt werden, gerade um Hilfestellung auch bei anderen Instituten der VC-Praxis zu gewähren.

\section{Zentrales Beispiel: Vesting-Regime in der GmbH}

\section{Logik von Anteilsverlust und korrespondierenden Erwerbsrechten}

Ihre Funktion als Finanzierungsinstrument erhalten Anteile in Vesting-Regimen durch eine Rückübertragungslogik. In den verbreiteten Vesting-Regimen mit Call-Option erhält der Gründer oder Mitarbeiter Geschäftsanteile, die einem Erwerbsrecht („Call-Option“) des Unternehmens, anderer Gesellschafter oder Dritter unterliegen („Call-Option-Anteile“). ${ }^{37}$ Er darf die CallOption-Anteile behalten, sofern kein festgelegter Tatbestand eintritt, der die Call-Optionsberechtigten zum Erwerb der Anteile durch „Call“ berechtigt („Leaver-Fall“). In Abhängigkeit von tatbestandlich ausdifferenzierten Umständen, kommt es zu einem unterschiedlich weitreichenden Verlust der CallOption-Anteile. ${ }^{38}$ Beispielsweise sind persönliche, schwere Verfehlungen, sowie eine außerordentliche Kündigung eines bestehenden Dienstvertrages aus wichtigem Grund, regelmäßig als „Bad-Leaver-Fall“ festgelegt, in dem der Gesellschafter alle Call-Option-Anteile verliert. Die Unmöglichkeit der weiteren Unternehmensförderung, insbesondere Tod, Arbeitsunfähigkeit oder Eigenkündigung eines Dienstvertrags durch den Gesellschafter selbst, sind typischerweise als "Good-Leaver-Fälle“ festgelegt, in dem nur ein Teil der CallOption-Anteile verloren geht und der „erdiente“ (auch: „gevestete") Teil beim Gesellschafter oder seinen Erben verbleibt.

36 Vgl. zu dieser zeitlichen Einordnung etwa Bank/Möllmann, aaO (Fn. 23), Teil 3 Rdn. 263. Umfragen unter Start-Ups ergeben einen Verbreitungsgrad von Vesting-Regimen von ca. 75 \%, vgl. SAmios/Arnold, aaO (Fn. 23), S. 153; die empirische Erhebung von KAPLAN/STRÖMBERg gehen von einem Verbreitungsgrad von noch $48 \%$ in den USA aus, Review of Economic Studies (2003) 70, 281, 292.

37 Zum Begriff Call-Option, vgl. Busche, Münchener Komm. z. BGB, 8. Aufl., 2018, Vor \145 Rdn. 70; Reichert/Weller, Münchener Komm. z. GmbHG, 3. Aufl., 2018, $\$ 15$ Rdn. $93 \mathrm{ff}$. Hier soll der Begriff unabhängig von seiner rechtlichen Ausgestaltung verwendet sein, zu dieser näher im folgenden Abschnitt.

38 Vgl. Kästle/Heuterkes, NZG 2005, 289, 290f; siehe auch die Formular-Verträge bei Weitnauer, aaO (Fn. 1), Anhang VI., Samios/Arnold, aaO (Fn. 23), S. $296 \mathrm{ff}$ und Bank/Möllmann, aaO (Fn. 23), Teil 3 S. 497 ff; daneben sind auch „grey“ Leaver-Fälle bekannt, in denen kein dinglicher Verlust eintritt, sondern lediglich die Gesellschafterrechte beschränkt werden. 
Zentral für den Umfang der im Good-Leaver-Fall verbleibenden Rechtsstellung ist mithin das „Erdienen“. Es wird zeitlich graduell bemessen, so dass mit Zeitablauf immer weniger Call-Option-Anteile vom Verlust im Leaver-Fall betroffen sind ${ }^{39}$ Regelmäßig besteht eine „Cliff-Periode“ von zumeist einem Jahr, vor deren Ablauf jeder Leaving-Fall, ob „good“ oder „bad“, zum vollständigen Verlust der Call-Option-Anteile führt. ${ }^{40}$ Die Call-Option-Anteile sind in der Regel nach vier bis sechs Jahren ohne Leaver-Fall vollständig erdient, so dass nach Ende der Vesting-Periode keine Erwerbsmöglichkeit durch Call-Option unter dem Vesting-Regime besteht.

Anzutreffen ist auch ein abgestuftes Regime für das Schicksal sonstiger Anteile, die nicht vom Verlust im Leaver-Fall betroffen oder dem Vesting-Regime überhaupt nicht unterworfen sind. Das sind insbesondere Anteile, die Gründern gehören, bevor VC-Investoren über Kapitalerhöhungen beteiligt werden (sog. „Founder-Anteile“) oder Anteile, die vor einer neuen Finanzierungsrunde bereits erdient sind. Im Leaver-Fall etwa können Gesellschafterrechte beschränkt oder mit den verbleibenden Call-Option-Anteilen auf einen Treuhänder übertragen werden. Daneben sind auch eine Abtretung und privilegierte Abfindungsregelungen möglich. ${ }^{41}$ Damit kann insbesondere der Bad-Leaver-Fall auch hinsichtlich der sonstigen Anteile den vollständigen Verlust der Mitgliedschaft zur Folge haben. Folgendes Schema - das tabellarisch auch in den geläufigen Gesellschafterabreden enthalten ist - soll den Zusammenhang von Call-Option-Anteilen und sonstigen Anteilen in Abhängigkeit vom Zeitpunkt des Leaver-Falles verdeutlichen:

Schema zum Ablauf einer nicht-linearen Vesting-Periode

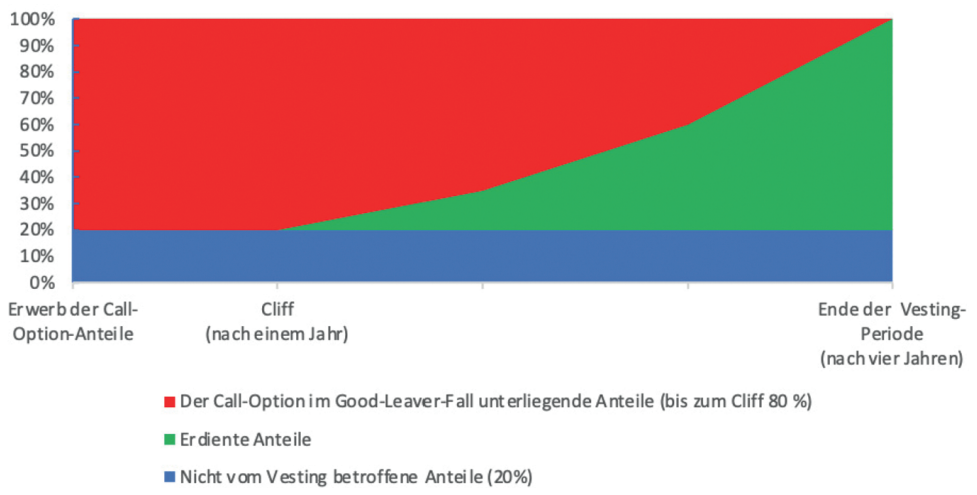

39 „Ansparen“, Bundesverband Deutsche Startups e.V., \#ESOPasap, 2020, S. 40; siehe auch die Muster bei Bank/Möllmann, aaO (Fn. 23), Teil 3 vor Rdn. 219; zur erfolgsabhängigen Bemessung, „Performance Vesting“, vgl. etwa Mackensen, in: Eilers/Koffka/Mackensen, Private Equity, 3. Aufl., 2018, S. 439.

40 Bank/Möllmann, aaO (Fn. 23), Teil 3 Rdn. 266.

41 Vgl. bei Bank/Möllmann, aaO (Fn. 23), Teil 3 Rdn. 241 ff. 
2. Ausgestaltung des Anteilsverlusts

\section{a) Satzungsregeln}

Das klassische Instrument zur Regelung des Anteilsverlusts ist die Zwangseinziehung nach $₫ 34 \mathrm{GmbHG}$. Allerdings wird bei der Einziehung der betroffene Anteil vernichtet, ${ }^{42}$ was im Leaver-Fall verhindert, den Anteil auf einen neuen Gesellschafter zu übertragen. Würde der gevestete Anteil im Leaver-Fall eingezogen, müsste für die Beteiligung neuer Gesellschafter eine aufwändige

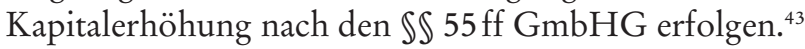

Denkbar ist weiterhin die Umsetzung des Vesting-Programms durch Zwangsabtretung. ${ }^{44}$ Im Gesellschaftsvertrag kann für Leaver-Fälle der Ausschluss per Gesellschafterbeschluss festgelegt und mit der Verfügungsberechtigung eines Geschäftsführers nach $\$ \mathbb{S} 182,185$ BGB gekoppelt werden. Dieser kann dann im Leaver-Fall die Abtretung anstelle des betroffenen Gesellschafters vornehmen. ${ }^{45}$ Statt der Vorabermächtigung der Geschäftsführung kann auch eine antizipierte Abtretung an einen zuvor benannten Begünstigten erfolgen, die auf den Gesellschaftsbeschluss bedingt ist.

\section{b) Regelung in einer Nebenabrede}

Satzungsregelungen zum Vesting haben sich in der Praxis nicht durchsetzen können. Vielfach wird stattdessen auf eine Gesellschafternebenabrede zur Satzung zurückgegriffen. Diese ist im Gegensatz zur Satzung nicht über das Handelsregister der Öffentlichkeit zugänglich. ${ }^{46}$ So ist in der Praxis die Herausbildung einer "Satzung hinter der Satzung" in Nebenabreden zu beobachten. Solche Nebenabreden - im Kontext des Venture Capital auch „Gesellschaftervereinbarungen (Shareholders' Agreements)" - sind als Substrat der Vertragsfreiheit grundsätzlich zulässig. ${ }^{47}$ Soweit die Übertragung von Geschäftsantei-

42 Vgl. etwa Sosnitza, in: Michalski/Heidinger/Leible/J. Schmidt, Komm. z. GmbHG, 3. Aufl., 2017, \34 Rdn. 121.

43 Vgl. zum Vesting durch Einziehung Kuntz, aaO (Fn. 1), 4. Teil, E., $\mathbb{\$}$ 2; Bank/MöllMANN, aaO (Fn. 23), Teil 3, Rdn. 229.

44 Dazu Göz/Kowalewski, NZG 2018, 136.

45 Die Vorabermächtigung kann aus wichtigem Grund widerrufen werden, vgl. MAIERReIMER, GmbHR 2017, 1325, 1331.

46 Vgl. etwa Wicke, DStR 2006, 1137; Hoffmann-Becking, ZGR 1994, 442, 445; GABRYSCH, in: Drygala/Wächter, Venture Capital, Beteiligungsverträge und „Unterkomplexitätsprobleme“, 2018, S. 64.

47 Vgl. etwa BGH NJW 1993, 2246; BGH NZG 2010, 988; K. SснмidT, Gesellschaftsrecht, 4. Aufl., 2002, \5 I 5.; Wicke, DStR 2006, 1137; eingehend Hoffmann-Becking, ZGR 1994, 442; zur historischen Entwicklung, Fleischer, RabelsZ 2018, 240, 254 ff; dazu 
len betroffen ist, unterliegen sie der notariellen Beurkundungspflicht, $\mathbb{} 15$ Abs. 3 und 4 GmbHG.

So findet sich in Nebenabreden, das dingliche Angebot der Gesellschafter, die Call-Option-Anteile auf die Gesellschaft oder auf Gesellschafter zurück zu übertragen („Reverse-Vesting“). ${ }^{48}$ Das Übertragungsangebot ist aufschiebend auf den Leaver-Fall bedingt, zumeist unbedingt und unwiderrufbar. ${ }^{49}$ Auf diese Weise ist die Effektivität des Leaver-Regimes sichergestellt, da dem Gesellschafter keine Obstruktionsmöglichkeit durch Widerruf des Angebots verbleibt. ${ }^{50}$

Die Feststellung eines Leaver-Falles wird zu Beweiszwecken regelmäßig von einem Gesellschafterbeschluss abhängig gemacht, bei dem nach $\mathbb{} 47$ Abs. 4 GmbHG der betroffene Gesellschafter von der Entscheidung ausgeschlossen ist. ${ }^{51}$ Es kann auch ein sonstiges Entscheidungsgremium bestimmt werden, insbesondere eine Runde von „Business-Angels“, Investoren und sonstigen „Brancheninsidern“. Wird ein Leaver-Fall festgestellt, können die in der Nebenabrede Begünstigten die vom Vesting betroffenen Anteile durch Ausübung der Call-Option erwerben. Das Übertragungsangebot wird durch Erklärung gegenüber dem betroffenen Gesellschafter angenommen, wobei nach $\mathbb{1 5}$ Abs. $3 \mathrm{GmbHG}$ die notarielle Form erforderlich ist und angesichts des sachenrechtlichen Bestimmtheitsgebots die zu übertragenden Anteile (etwa mithilfe einer Vesting-Tabelle) genau zu bezeichnen sind. Der Zugang der Annahmeerklärung ist nach $\$ 151$ Satz 1 BGB abdingbar.

Eine lediglich schuldrechtliche Verpflichtung zur Rückübertragung der Optionsanteile ist ebenfalls möglich, gibt den Berechtigten aber eine wesentlich schwächere Stellung und ist daher Zeichen einer starken Verhandlungsposition des Gründers oder Mitarbeiters. Verstößt der Gesellschafter gegen diese in der Nebenabrede vereinbarte schuldrechtliche Pflicht die sonstigen Anteile zu übertragen, kann allerdings ein wichtiger Grund zur Einziehung der Geschäftsanteile bestehen. ${ }^{52}$

auch NOACK, NZG 2013, 281; DERs., Gesellschaftervereinbarungen bei Kapitalgesellschaften, 1994; rechtsvergleichend Laimer/Perathoner, Gesellschaftsrechtliche Nebenvereinbarungen in Europa, 2013; KunTZ, aaO (Fn. 1), S. $18 \mathrm{ff}$.

48 Vgl. etwa Kästle/Heuterkes, NZG 2005, 289, 290 ff.

49 Zum Widerrufsvorbehalt zeitlich weit gestreckten Verträgen über die Übertragung von GmbH-Geschäftsanteilen, BGH DNotZ 1991, 917 f.

50 SAmios/Arnold, aaO (Fn. 23), S. 149; zum ansonsten bestehenden „Lästigkeitspotential“ und der Erzwingbarkeit von Abfindungen siehe auch Stenzel, aaO (Fn. 23), Rdn. 611.

51 Vgl. etwa Gehrlein, WM 2019, 1, 2.

52 So etwa Hoffmann-Becking, ZGR 1994, 442, 461. 


\section{c) Rechtsschutz}

Bei der herkömmlichen Ausgestaltung der Call-Option als unbedingtes und unwiderrufbares Angebot steht der Gerichtsweg nur gegen den Beschluss zur Ausübung der Call-Option offen. Anfechtung und Nichtigkeitsfeststellungsklage wirken dabei noch nicht dinglich auf die Inhaberschaft am Geschäftsanteil, so dass eine Kondiktionsklage die einzig gangbare Verteidigungsmöglichkeit ist. ${ }^{53}$ Denn der Beschluss über die Ausübung der Call-Option ist verschieden von der Ausübung der Call-Option selbst. Auf Grund der Aufwertung der Gesellschafterlisten durch das MoMiG in $\$ 16 \mathrm{GmbHG},{ }^{54}$ ist ein inzwischen verbreiteter, informeller Rechtsbehelf der Widerspruch gegenüber dem bei der rechtsgeschäftlichen Veräußerung von Geschäftsanteilen mitwirkungspflichtigen Notar. Dieser ist zwar nicht an Weisungen der Gesellschafter gebunden, muss allerdings prüfen, ob die dem Übertragungsangebot zu Grunde liegende aufschiebende Bedingung eingetreten ist bevor er die Liste zum Handelsregister einreicht. ${ }^{55}$

\section{d) Vesting als „umgekehrtes Anwartschaftsrecht"}

Obwohl es auf wirtschaftlicher Ebene um die Beteiligung geht, ist die rechtstechnische Umsetzung unter deutschem Recht nach dem Vorstehenden über ein Verlustprogramm hinsichtlich zunächst vollumfänglich übertragener Anteile ausgestaltet. Eine stufenweise Beteiligung nach Maßgabe des Erdienens würde wiederholt notarisierungspflichtige Vorgänge voraussetzen. ${ }^{56}$ Auch können durch eine sofortige umfängliche Übertragung Lohnsteuervorteile erzielt werden, wenn man davon ausgeht, dass die Anteile im Wert steigen. ${ }^{57}$ Die praktisch gewollte Entlohnung von Gründern und Mitarbeitern wird also unter sich laufend verringerndem Entziehungsvorbehalt durchgeführt.

Rechtsinstitutionell liegt damit ein Vergleich des Vesting mit dem sachenrechtlichen Anwartschaftsrecht nahe. Denn der Erwerber eines Anwartschaftsrechts hat ebenfalls eine hybride Position, zwischen dinglicher Berechtigung und schuldrechtlicher Sicherung. Nach überkommener Definition setzt ein

53 Zum sofort dinglich wirksamen Vorgehen gegen Einziehungsbeschlüsse vgl. STROHN, Münchener Komm. z. GmbHG, 3. Aufl., 2018, $\$ 34$ Rdn. 83 ff.

54 Vgl. zu den Auswirkungen des MoMiG, etwa MaYer, MittBayNot 2014, 24 und 114.

55 Vgl. Eвbing, in: Michalski/Heidinger/Leible/J. Schmidt, Komm. z. GmbHG, 3. Aufl., 2017, 16 Rdn. 34; Heidinger, Münchener Komm. z. GmbHG, 3. Aufl., 2019, $\mathbb{} 40$ Rdn. 212.

56 Vgl. Gabrysch, aaO (Fn. 46), S. 62 f, der auch auf die Lösung im US-Recht hinweist.

57 Siehe die Nachweise in Fn. 29; zuletzt zu negativen Liquidationspräferenzen KunTz/ ENGELHARDT, ZGR 2021, 348. 
Anwartschaftsrecht voraus, dass vom Entstehungstatbestand eines Rechts schon so viele Erfordernisse erfüllt sind, dass der Veräußerer die Rechtsposition des Erwerbers nicht mehr durch einseitige Erklärung zerstören kann..$^{58}$ Die mit Zeitablauf erdienten Geschäftsanteile sind gerade nicht mehr wegen Umständen, die einen Good-Leaver-Fall begründen, zurückzuübertragen. Ihr Verlust ist nur wegen Umständen möglich, die einen Bad-Leaver-Fall begründen - und diese Umstände hat der Gesellschafter allein in der Hand. Insoweit hat kein Dritter, analog zum Veräußerer, eine Rückholbefugnis über die erdienten Anteile. Allein für noch nicht erdiente Anteile kann sich ein GoodLeaver-Fall auswirken. Nach der Leitidee des Erdienens ist die rechtliche Perspektive des Vesting also - im Gegensatz zur Anwartschaft - gerade nicht der Erwerb einer Position, sondern ihr Verlust, weshalb es in der Perspektive des Begünstigten vergleichbar zum umgekehrten Anwartschaftsrecht erscheint. ${ }^{59}$

\section{Ausgestaltung der Entschädigung}

In Bad-Leaver-Fällen und Leaver-Fällen vor Erreichen des Cliffs stehen alle Call-Option-Anteile zur Disposition, in Good-Leaver-Fällen nach Erreichen des Cliffs nur die noch nicht erdienten Call-Option-Anteile. Wird die CallOption wahrgenommen, so verliert der Gesellschafter die betroffenen CallOption-Anteile an den Call-Option-Berechtigten. Die sonstigen Anteile, insbesondere die Founder-Anteile, gehen in Bad-Leaver-Fällen regelmäßig ebenfalls über - sei es durch Zwangsabtretung, Einziehung, aufschiebend bedingte Übertragung oder separate Übertragung. Die Entschädigung für den Verlust der nicht erdienten Call-Option-Anteile ist regelmäßig auf ihren Nennwert, also meist einen Euro festgesetzt. Eine Abfindung zu einem höheren, etwa Buch- oder Verkehrswert, kann insbesondere für eine junge Gesellschaft im Leaver-Fall erhebliche Liquiditätsprobleme bedeuten. ${ }^{60}$ Anzutreffen sind allerdings durchaus Fälle, in denen eine Abfindung auch zum Verkehrswert vereinbart ist, insbesondere bezüglich der zum Zeitpunkt des Leaver-Falles erdienten Anteile und Founder-Anteile, sofern diese neben den Call-Option-Anteilen übergehen sollen.

58 BGH NJW 1958, 1133; Armgardt, AcP 206 (2006), 654 f; Oechsler, Münchener Komm. z. BGB, 8. Aufl., 2020, \$929 Rdn. 18; kritisch zum Anwartschaftsrecht an sich Mülbert, AcP 214 (2014), 309.

59 Siehe auch zum umgekehrten Vesting, BLoß, GmbHR 2016, 104, 110.

60 Vgl. etwa Samios/Arnold, aaO (Fn. 23), S. 151; Bank/Möllmann, aaO (Fn. 23), Teil 3, Art. 5 Rdn. $236 \mathrm{ff}$. 


\section{Kautelarpraxis der Wagniskapitalfinanzierung}

\section{1. „Beteiligungs-Menüs“ aus Musterklauseln}

Mit der komplexen Interessenlage und Beteiligungslogik von Vesting-Regimen ist, wie bei den übrigen Instrumenten von Wagniskapitalbeteiligungen, ihre Genese eng verknüpft. Sie sind Produkt einer privaten, internationalen und pragmatischen Investitionspraxis. Typischerweise sind sie Element eines umfassenden Investitionsprogramms in Beteiligungsverträgen und Gesellschafterabreden, die auch Bedingungen zu Ablauf, Art und Zeitpunkt der Investitionsleistung enthalten, daneben Regelungen zur synallagmatischen Übertragung von Geschäftsanteilen, sowie VC-spezifische Regeln zu Liquidationspräferenzen, Reporting-Pflichten, Garantien von Gesellschaft und Gründern oder Tagund Drag-Along-Klauseln. ${ }^{61}$ Diese umfassenden Beteiligungsprogramme sind durch die oben geschilderten, spezifischen Bedürfnisse der VC-Finanzierung geprägt.

Wie im Finanzierungsrecht insgesamt, ist dabei private Normierung durch die Kautelarjurisprudenz dominant. ${ }^{62}$ Die Praxis ist hier ausgehend vom konkreten Gestaltungsbedarf im Markt den staatlichen Regelsetzern vorausgeeilt. Der weite Gestaltungsspielraum der VC-Praxis folgt aus dem überwiegend dispositiven Charakter des Gesellschaftsrechts, ${ }^{63}$ wobei freilich auch dessen Lü-

61 Siehe dazu etwa Weitnauer, aaO (Fn. 1), Teil F Rdn. $118 \mathrm{ff}$; Bank/Möllmann, aaO (Fn. 23), Teil 2; siehe auch den Beispielsbeteiligungsvertrag bei SAmios/Arnold, aaO (Fn. 23), S. 92 ff; zu Tag- und Drag-Along-Klauseln, siehe Fleischer/Schneider, DB 2012, 961 und Bank/Möllmann, aaO (Fn. 23), Teil 3, Art. 6, 19, 20.

62 Dazu Choi/Gulati, The Emory Law Journal, 53, 929-996; Dies., 104 Mich. L. Rev. 1129 (2006), 1130; vgl. etwa zur Bedeutung der standardisierten Unternehmenskreditverträge der Loan Market Association, Renner, in: Grundmann, Bankvertragsrecht, Bd. 2, 2020, Vierter Teil Rdn. $346 \mathrm{ff}$; zu Begriff und Erscheinungsformen nichtstaatlichen Rechts, Hellgardt, RabelsZ 2018, 657 ff; zur Selbstregulierung allgemein und umfassend,

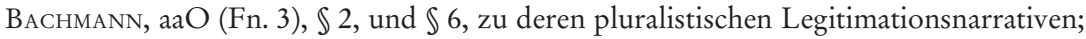
grundlegend im 20. Jahrhundert GroßMAnN-Doerth, aaO (Fn. 5); zur Bedeutung internationaler Klauselwerke, GrundmanN, in Ebenroth/Boujong/Joost/Strohn, Komm. z. HGB, 4. Aufl., 2020, Vorbemerkung Deutsches Recht der Handelsgeschäfte im Kontext des Unionsrechts sowie des internationalen Rechts, Rdn. 77 ff; Kuntz, aaO (Fn. 1), S. $350 \mathrm{ff}$.

63 Vgl. Fleischer, RabelsZ 2018, 240, 261 ff; Merkt, aaO (Fn. 4), S. 167 ff; Windbichler, $\mathrm{aaO}$ (Fn. 31), $\$ 2$ Rdn. 20, $\$ 4$ Rdn. 1 ff; Treeck, Das Ausscheiden aus der Doppelgesellschaft, 1968, S. 5, $16 \mathrm{ff}$; vgl. etwa zur Einmann-AG Bachmann, NZG 2001, 961. Vgl. K. Schmidt, aaO (Fn. 47), $\mathbb{} 2$ I 2, 3; Windiichler, aaO (Fn. 31), $\$ 3$ Rdn. 4, 30; siehe auch bei Kuntz, aaO (Fn. 1), Einleitung, B. und zur Typenlehre, dem Numerus Clausus des Gesellschaftsrechts und zur Institutionentheorie, 2. Teil, A. $\$ 1$, 3. f; zur Bemühung um die Allgemeinen Lehren des Gesellschaftsrechts, vgl. m.w.N. Grundmann, Der Treuhandvertrag, 1997, S. $122 \mathrm{f}$. 
cken, wie beim Gesellschafterausschluss, gerade ein treibender Faktor für Innovation in der Kautelarpraxis sind. ${ }^{64}$

Die oben nachgezeichnete wirtschaftliche Logik von VC-Beteiligungen, wie insbesondere die Korrespondenzbeziehung von Anteilsverlust und Erwerbsrechten zu Zwecken der Investitionssicherung sowie Mitarbeitermotivierung, stammt aus dem angelsächsischen Rechtsraum und wurde zunächst durch einige wenige Rechtsanwaltskanzleien, Fonds und Inkubatoren ${ }^{65}$ in Deutschland rezipiert. ${ }^{66}$ Die gegenwärtige Verfasstheit der VC-Beteiligungsverträge in Deutschland folgt aus der ursprünglich angloamerikanischen Prägung der Investoren und spiegelt auch die Internationalität der Gründer wider. ${ }^{67}$ Die USAmerikanischen Verträge stellen immer noch den internationalen „Gold-Standard" für VC-Beteiligungen dar, ${ }^{68}$ was auch in den wichtigsten Formularhandbüchern für VC-Beteiligungen betont wird. ${ }^{69}$

Die Musterklauseln zentraler Venture Capital-Akteure werden typischerweise ohne Veränderung der beschriebenen Strukturlogik verwendet; Gegenstand rechtlicher wie unternehmerischer Modifikationen sind dann vor allem die Wahl konkreter Klauseln und Ausschlussgründe (Leaver-Fälle) sowie die Dauer der Vesting-Periode und die Abfindung. Empirische Untersuchungen haben trotz der denkbaren Vielfalt an Kombinationsmöglichkeiten von Klauseln ergeben, dass es zu einer erheblichen Standardisierung und Spezialisierung gekommen ist. Gewählt wird tatsächlich aus einem sehr begrenzen „Menü“ an Klauseln. Anpassungen im Einzelfall haben im Verhältnis zur grundlegenden Logik lediglich Detailcharakter. ${ }^{70}$

64 Siehe zu alledem sogleich, unter V. 1.; vgl. zur Kritik am Fehlen einer gesetzlichen Regelung zum Gesellschafterausschluss, GeHrlein, NJW 2005, 1969; Götz/Kowalewski, NZG 2018, 1369; Grunewald, aaO (Fn. 23), S. 125.

65 Ein Inkubator - metaphorisch angelehnt an den Brutkasten - unterstützt Gründer mit Finanzmitteln und Know-How im Gegenzug für die Einräumung von Kontrollrechten über die Gesellschaft - wie insbesondere Vesting-Regime; vgl. umfassend schon früh OECD, Business Incubation: International Case Studies, 1999.

66 Zur Rolle von Rechtsanwaltskanzleien für die Standardsetzung im Venture-Capital vgl. Bernstein, 74 Oreg. L. Rev., 239 (1995); Vronsky, in: Cumming, The Oxford Handbook of Venture Capital, 2012, S. 602, 609; mit einem empirischen Vergleich der US und Deutschen Praxis, Antonczyk/Breuer/Brettel, FB 2008, 225; zu weiteren Bestrebungen der Standardisierung siehe etwa die Vorlagen des German Standards Setting Institute, abrufbar unter: standardsinstitute.de/standarddokumentel (letzter Zugriff: 22.8.2021).

67 SAmios/Arnold, aaO (Fn. 23), S. 83 ff; siehe auch: https://www.vc-magazin.de/venturecapital/beteiligungsvertraege-im-venture-capital-sektor/ (letzter Zugriff: 22.8.2021).

68 So Bengtsson, aaO (Fn. 1), S. 478, 479.

69 Etwa Weitnauer, aaO (Fn. 1), Teil A Rdn. 59 - 76.

70 Dies belegt die empirische Untersuchung von BENGTSSON/BERNHARDT anhand von über 4500 VC-Beteiligungsverträgen in den USA, Journal of Economics \& Management Stra- 
Dennoch sind Anpassungsunschärfen in Bezug auf das Vertragsganze denkbar. Hinzu kommen Fallstricke in der Übersetzung oder englischsprachigen Verwendung der Musterverträge und die Schaffung eigener Begrifflichkeiten, wofür die hier bereits thematisierten „Call-Optionen“, „Leaver-Fälle“, „CliffPerioden" und das "Vesting" an sich Beispiel geben. Derartige Unschärfen werden pragmatisch in Kauf genommen, da die Verwendung branchenüblicher Muster erhebliche Transaktionskostenvorteile bietet und gerade auf Agilität ${ }^{71}$ ausgerichtete Startups nicht hinreichend Zeit und Ressourcen verwenden können, um selbst umfassende Finanzierungs- und Mitarbeiterbeteiligungskonzepte zu entwerfen. VC-Investoren können die Komplexität der Investitions-, Anreiz- und Sicherungslogiken durch Spezialisierung auf wenige Typen von Klauseln ausgleichen. Die Kostenvorteile der Typisierung sollen so die Überspannungen von Klauseln im Einzelfall kompensieren. ${ }^{72}$ Daneben versprechen sich die Parteien durch Nutzung etablierter Standards auch Rechts- und Verhaltenssicherheit. ${ }^{73}$ Diese Motivlage dürfte wohl charakteristisch für die private Erstellung dispositiven Rechts sein. ${ }^{74}$ Sie äußert sich durch eine weitgehende Akzeptanz im Markt. ${ }^{75}$

tegy, 2014-06, Vol.23 (2), p. 396-426; zur typisierung von VC-Verträgen in den USA anhand einer Probe von über 200 Verträgen vgl. Kaplan/Strömberg, Review of Economic Studies (2003) 70, 281-315; verfehlt dürfte es daher sein, pauschal von seitens der Investoren vorgegebene standardisierte Vertragsmuster zu sprechen, wie etwa WeITNAUER, aaO (Fn. 1), Teil F. Rdn. 108 und WINKLER, Rechtsfragen der Venture Capital-Finanzierung, 2004, S. $94 \mathrm{ff}$.

71 Beim betriebswirtschaftlichen Konzept der Agilität stehen kurzschrittiges Handeln und Dezentralität im Vordergrund, vgl. zum (weit belegbaren) Begriff ursprünglich das Agile Manifesto (abrufbar unter: agilemanifesto.org, letzter Zugriff: 22.8.2021); sowie GLOGER/RÖSNER, Selbstorganisation braucht Führung: Die einfachen Geheimnisse agilen Managements, 2. Aufl., 2017, S. $19 \mathrm{ff}$.

72 Zum Kalkül der Vertragserstellungskosten gegenüber den potentiellen Streitkosten, PosNER, The Law and Economics of Contract Interpretation, 3 Tex. L. Rev. 2005, 1581, 1584.

73 Dazu Bengtsson/Bernhardt, Journal of economics \& management strategy, 2014-06, Vol.23 (2), p. 396-426; allgemein zur „Stickiness“ dispositiven Rechts Ben-SHaHar/PotTow, Florida State University Law Review 33 (2006), 651; McDonnell, SMU L.R. 60 (2007), 383.

$74 \mathrm{Zu}$ den ökonomischen Hintergründen der Standardisierung durch Vertragsmuster vgl. KuntZ, aaO (Fn. 1), S. 363 ff; zur Transaktionskostenersparnis durch dispositives Recht, siehe Möslein, Dispositives Recht, 2011, S. $302 \mathrm{ff}$.

75 Zur Akzeptanz als Legitimitätsgrund, vgl. Merkt, aaO (Fn. 4), S. 167, 177; allgemein zu Legitimationsmodellen Bachmann, in: Bumke/Röthel, Privates Recht, 2012, S. $207 \mathrm{ff}$, insbes. S. $215 \mathrm{ff}$. 


\section{Einordnung als Handelsbrauch}

Die beschriebene Entwicklung von Marktstandards wirft die Frage auf, wie die Kautelarpraxis bei VC-Beteiligungen einzuordnen ist - und welche Auswirkungen sie auf die rechtliche Überprüfbarkeit der jeweiligen Beteiligungsabreden und insbesondere der Verlustprogramme hat. Die Frage, wie Verhaltenserwartungen zu berücksichtigen sind, die im Zusammenhang mit den sozialen Rollen und ökonomischen Intentionen bei VC-Beteiligungen aktuell werden und im relevanten Verkehrskreis auf Konsens stoßen ist für den vorliegenden Beitrag zentral.

Bisweilen wird sogar vertreten, dass eine rechtliche Überprüfbarkeit von VCBeteiligungen vollständig ausscheiden müsse. ${ }^{76}$ In diese Richtung geht auch der im US-Recht prominente Ansatz von Choi/Gulati, standardisierte Verträge zwischen Unternehmern, in denen anders als im prototypischen Fall des AGB-Rechts regelmäßig gerade keine strukturelle Asymmetrie zwischen den Parteien vorzufinden ist („boilerplate contracts between sophisticated parties“), wie Gesetze zu behandeln und die einzelfallspezifischen Parteiinteressen auszublenden. ${ }^{77}$ Im deutschen Recht wäre dies bislang allenfalls vergleichbar mit der AGB-rechtlichen Privilegierung der VOB/B in $\$ 310$ Abs. 1 Satz 3 BGB, die sich allerdings aus einer Einbeziehung auch staatlicher Stellen in die Genese und gerade dadurch eine besondere personelle Legitimität und Interessenausgleich begründet. $^{78}$

Indes prägen wie gezeigt primär wirtschaftliche - und nicht rechtliche oder gemeinwohlorientierte - Belange die VC-Praxis, die zum beschriebenen Funktionswandel von Geschäftsanteilen führen. Hinzu kommt, dass rechtskulturelle Übertragungen aus dem US-Recht in deutsche Beteiligungsverträge fehleranfällig sind, so dass schon deshalb auf eine Wirksamkeitskontrolle der Beteiligungsbedingungen nicht ganz verzichtet werden kann. Auch die deutsche Dogmatik zu Verkehrssitten im Handelsrecht steht einer Übertragung des genannten Ansatzes zu „boilerplate contracts“ entgegen.

Die rechtliche Bedeutung von Branchenstandards als normative Leitmedien ist im deutschen Recht allerdings noch nicht abschließend geklärt. Die Behandlung des „selbstgeschaffenen Rechts der Wirtschaft“ Anfang des 20. Jahrhunderts ist fragmentarisch geblieben, erfährt freilich durch Phänomene wie der Corporate Codes of Conduct und der Corporate Governance Codices eine Renaissance. ${ }^{79}$

76 Vgl. etwa Samios/Arnold, aaO (Fn. 23), S. 147.

77 Choi/Gulati, 104 Mich. L. Rev. 1129 (2006), 1130; mit einem starken formalistischen Ansatz auch Schwartz/ScotT, 119 Yale L. J. (2010), 926.

78 BGHZ 86, 135; dazu RyLl, NZBau 2018, 187, 188.

79 Vgl. Merkt, aaO (Fn. 4), S. 171 ff; skeptisch Teubner, Standards und Direktiven in Generalklauseln, 1970, S. 32 f. 
Richtig dürfte sein, die Musterklauseln bei VC-Beteiligungen als Handelsbrauch zu werten und als solchen in der rechtlichen Würdigung zu berücksichtigen. ${ }^{80}$

Unklarheit besteht freilich schon über den Geltungsgrund des Handelsbrauchs, wobei die Begründungsansätze in der Literatur einer normativen Theorie zuneigen. ${ }^{81}$ Die Geltung von Handelsbräuchen soll allerdings nicht rein rechtlich begründet sein, sondern eher reziprok, mittels eines rechtssoziologischen Befundes, „weil der Verkehr dies erwarte und erwarten dürfe“ .82

Für Handelsbräuche konstitutiv ist daher eine gleichmäßige, einheitliche und freiwillige Übung der beteiligten Kreise für vergleichbare Geschäftsvorfälle über einen angemessenen Zeitraum hinweg. ${ }^{83}$ Diesen Anforderungen genügen die zu Beteiligungs-Menüs komponierbaren Musterklauseln der Beteiligungsinstrumente des Venture Capital, die immerhin schon seit mehreren Jahrzehnten einvernehmlich von Investoren, Gründern und Kanzleien verwendet werden. Durch sie erfolgt der Paradigmenwechsel zum Einsatz von Geschäftsanteilen als Finanzierungs- und Vergütungsinstrument und damit auch eine Verschiebung der Ausschlussproblematik - weg von einer reinen mitgliedschaftlichen Binnenfrage hin an die Schnittstelle vom Unternehmen zum Markt. ${ }^{84}$ Daher passt auch die Einordnung von VC-Beteiligungsregelungen als Frage auch des Handelsrechts.

Eine Verstetigung zu Gewohnheitsrecht, wie etwa im Beispiel des kaufmännischen Bestätigungsschreibens, ${ }^{85}$ ist hingegen nicht anzunehmen. Gewohnheitsrecht ist nur mit äußerster Zurückhaltung festzustellen, zumal private Normen anders als demokratisch zustande gekommene Gesetzestexte eine nur rudimentäre Gerechtigkeitsgewähr zusichern können, insbesondere Gemein-

80 Zur Bedeutung des Handelsbrauchs allgemein, Canaris, Handelsrecht, 24. Aufl., 2006, \22; zur rechtlichen Wirkung BachmanN, aaO (Fn. 3), $\mathbb{1 0}$ B.; vgl. zum Zusammenhang von Handelsbrauch und Klauselwerken, Maultzsch, Münchener Komm. z. HGB, 5. Aufl., 2021, $\ 343$ Rdn. 24; mit einer kritischen Systematisierung, Hellwege, AcP 214 (2014), 853.

81 Mit umfassendem Überblick zum Streitstand, Hellwege, AcP 214 (2014) 853, 854 ff. Mit einer Abgrenzung zum Gewohnheitsrecht, K. Sснміdт, Handelsrecht, 6. Aufl., 2014, $\mathbb{} 1$ III Rdn. 48f. Zur Normativität der rechtswissenschaftlichen Methode MetZger, Extra legem, intra ius: Allgemeine Rechtsgrundsätze im Europäischen Privatrecht, 2009, S. $48 \mathrm{ff}$.

82 Vgl. Hellwege, AcP 214 (2014), 853, 855f; Prütting/Weller, Handels- und Gesellschaftsrecht, 10. Aufl., 2020, Rdn. 32.

83 So auch BGH NJW 1994, 659; 2001, 2465; WM 1984, 1002; NJW 2018, 1959 Rdn. 30.

84 Grundlegend zur Dialektik von Markt und Organisation Williamson, Markets and Hierarchies, 1975; auch Powell, 'Neither Market nor Hierarchy - Network Forms of Organization', 12 Research in Organizational Behaviour, 1990, 295-336.

Dazu K. Schмidt, aaO (Fn. 81), \$19 II Rn 66-71. 
wohlbelange ausblenden. ${ }^{86}$ Erforderlich ist daher eine erhebliche „normative Verfestigung “87 der Verkehrssitte, die angesichts der relativen Neuartigkeit von VC-Beteiligungsprogrammen, ihrer letztendlich umfassenden Verhandelbarkeit im Einzelfall und der häufig lediglich ökonomischen Verwendungsmotivation zur Kosteneinsparung ausscheiden dürfte.

Handelsbräuche typisieren Verhaltenserwartungen. ${ }^{88}$ Rechtsfolge von Handelsbräuchen ist daher nach der h.M. ein Einfluss auf die Vertragsauslegung nach $\int S 157 \mathrm{BGB}, 346 \mathrm{HGB} .^{89}$ Als Transformationsnormen führen diese Vorschriften zur Geltung der Verkehrssitte zwischen den Parteien, unabhängig von deren Kenntnis, gar Kennenmüssen, der Verkehrssitte. ${ }^{90}$ Im Folgenden wird zu zeigen sein, dass die VC-Kautelarpraxis im Bereich des Gesellschafterausschlusses allerdings eine noch weitreichendere Wirkung des Topos Handelsbrauch rechtfertigt.

\section{Wirtschaftssoziologischer Ansatz zur Hinauskündigungsrechtsprechung}

Auf herkömmliche Ausschlussklauseln in der Satzung einer Gesellschaft ist die Hinauskündigungsrechtsprechung anzuwenden. Auf Grund des normativen Funktionswandels von Geschäftsanteilen durch die VC-Kautelarpraxis, hin zu Finanzierungs- und Vergütungsinstrumenten, ist die Anwendung der Hinauskündigungsrechtsprechung an die wirtschaftlichen Verhältnisse anzupassen.

\section{Offene Dogmatik des Gesellschafterausschlusses}

Die Regelung zum Anteilsverlust unter Vesting-Regimen kann vor Hintergrund der allgemeinen Dogmatik des Gesellschafterausschlusses zu Zweifeln an der Wirksamkeit von Vesting-Regimen führen. ${ }^{91}$ Ausschluss- und Abfindungsregelungen für Gesellschafter wurden von RG und BGH schon sehr unterschiedlich gewürdigt. Neben der allgemeinen Ausschlussklage sind vor al-

86 Dazu Bachmann, aaO (Fn. 75), S. 207 ff, insbes. S. $212 \mathrm{ff.}$

87 K. Schmidt, aaO (Fn. 81), $\$ 1$ III Rdn. 49.

88 K. Sснмidt, aаO (Fn. 81), $\$ 1$ III Rdn. 48f; die stärkere Berücksichtigung normativer Verkehrserwartungen befindet sich derzeit im Vordringen, vgl. etwa RENNER, AcP (213) 2013, 677; Grünberger, AcP (218) 2018, 213; WielsCh, RW 1/2019, 84, 86 f.

89 CANARIS, aaO (Fn. 80), $\ 22$ Rdn. 11; zur Unterordnung gegenüber $\$ 242 \mathrm{BGB}$ vgl. BUSCHE, aaO (Fn. 37), $₫ 157$ BGB Rdn. 17.

90 Zum fehlendem subjektiven Element OetKer, Handelsrecht, 8. Aufl., 2019, $\$ 7$ Rdn. 52; zur Einordnung als Transformationsnorm, Jung, Handelsrecht, 12. Aufl., 2019, $\mathbb{} 34$ Rdn. 12; siehe auch Hellwege, AcP 214 (2014), 853, $863 \mathrm{ff.}$

91 Siehe etwa auch SAmios/Arnold, aaO (Fn. 23), S. 151. Mit einem eher pauschalen Vorwurf vgl. Bundesverband Deutsche Startups e.V., \#ESOPasap, 2020, 41. 
lem Satzungsregelungen zum Gesellschafterausschluss Gegenstand einer längeren Rechtsprechungshistorie. ${ }^{92}$ Diese nahm ihren Ausgangspunkt im Recht der Personengesellschaften, ist wegen der personalistischen Ausgestaltbarkeit der $\mathrm{GmbH}$ freilich auf diese übertragbar. ${ }^{93}$

Zunächst wurde bereits die Zulässigkeit des Gesellschafterausschlusses an sich verschieden hergeleitet, zum einen aus den Grundsätzen des Handelsrechts und Treuepflichten, ${ }^{94}$ zum anderen aus der allgemeinen Vertragsfreiheit. ${ }^{95}$ Die Wirksamkeit des Ausschlusses bei der Ausschlussklage soll am wichtigen Grund, ${ }^{96}$ bei Satzungsregeln am sachlichen Grund und nach der sog. Hinauskündigungsrechtsprechung ausnahmsweise sogar lediglich „besonderen Umständen des Einzelfalls" zu messen sein. ${ }^{97}$ Satzungsregeln werden dabei am Maßstab der Sittenwidrigkeit überprüft. ${ }^{98}$ Zuvor wurden freilich verschiedene andere Leitlinien angelegt, von „den Grenzen der Parteiautonomie“, 99 über eine Kernbereichslehre der Gesellschafterstellung, ${ }^{100}$ bis zum Treuegedanken des Personen- und Kapitalgesellschaftsrechts. ${ }^{101}$ Auch für die Abfindungsregelungen ergibt sich ein uneinheitliches Bild. ${ }^{102} \mathrm{Im}$ Endergebnis steht auch unter der Hinauskündigungsrechtsprechung für die Frage der Wirksamkeit von Ausschluss- und Abfindungsklauseln eine Abwägung der Interessen des Gesellschafters, der Gesellschaft und gegebenenfalls der Gläubiger des Gesellschafters. ${ }^{103}$

92 Vgl. zur Kritik Gehrlein, NJW 2005, 1969; Schwab, DStR 2012, 707; Römermann, NZG 2010, 96f; zur Kritik siehe etwa Mülsch/Penzel, ZIP 2004, 1987, 1989 f.

93 So auch Ulmer/Habersack, in: Habersack/Casper/Löbbe, Großkomm. z. GmbHG, 3. Aufl., Bd. 2, 2020, $\$ 34$ Rdn. 41 a f; für Anwendung auch auf kapitalistisch ausgestaltete GmbH Strohn, aaO (Fn. 53), $\$ 34$ GmbHG Rdn. 104.

94 BGH NJW 1953, 780, 781; A. Hueck, DB 1953, 776; m. w. N. Strohn, aaO (Fn. 53), \$34 GmbHG Rdn. 103.

95 RGZ 169, 330, 334; mit umfassendem Überblick GeHrLein, NJW 2005, 1969.

96 BGH NJW 1953, 780, 781; SCHWAB, DStR 2012, 707, 709; m. w. N. WindiICHLER, aaO (Fn. 31), $\$ 22 \mathrm{Rdn} .26 \mathrm{f}$.

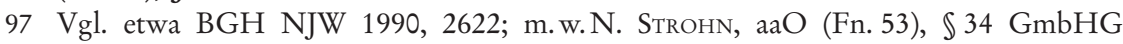
Rdn. 141; Armbrüster, Münchener Komm. z. BGB, 8. Aufl., 2018, 138 Rdn. 83.

98 BGH NJW 1981, 2565; BGH NJW 1989, 834; GeHrlein, WM 2019, $1,2$.

99 BGH NJW 1977, 1292; früh dazu Schilling, ZGR 1979, 419.

100 BGH, Urt. v. 19.10.2009 - II ZR 240/08, BGHZ 183, 1 = NJW 2010, 65 Rdn. 16 Sanieren oder Ausscheiden; anklingend noch bei GeHrLeIn, NJW 2005, 1969, 1973. Hierher gehört auch das Bild des Damoklesschwerts, vgl. BGHZ 81, 263, $266 \mathrm{ff}$.

101 Dazu Schilling, ZGR 1979, 419; mit einer Übersicht zu den weiteren Meinungen in der Literatur GeHrLein, NJW 2005, 1969, $1971 \mathrm{f}$.

102 Vgl. BGH WM 2014, 1343 für die Heranziehung der Sittenwidrigkeit; BGH NJW 1992, 892, $895 \mathrm{f}$ für das allgemeine Gleichbehandlungsgebot aus $\$ 243$ Abs. 2 AktG; BGH NJW 1985, 192, 193; NJW 1989, 3272 für eine Vereitelung des Kündigungsrechts; Ulmer/Habersack, aaO (Fn. 93), Anh. $\$ 34$ GmbHG Rdn. 108 für die Gläubigerbenachteiligung.

103 Vgl. so auch im Ergebnis GeHrLein, WM 2019, 1. 
Die für Satzungsregelungen entwickelte Hinauskündigungsrechtsprechung übertrug der $\mathrm{BGH}$ ohne größeren Begründungsaufwand im prominenten Mediamarkt-Saturn-Fall auf die Ausschlussvereinbarung in Nebenabreden. ${ }^{104}$ Dieser Übertragung auf Nebenabreden ist grundsätzlich zuzustimmen, da dort die Privatautonomie noch stärker zum Tragen kommt als bei der Modifikation bestehender Satzungsvereinbarungen oder gar bei vollständigem Fehlen von Vereinbarungen. Daher muss auch nicht der strengere Maßstab des wichtigen Grundes wie bei der Ausschlussklage gelten. Die Wechselhaftigkeit der Hinauskündigungsrechtsprechung ${ }^{105}$ legt allerdings auch ihre Erweiterungsfähigkeit nahe - und gibt Raum für neue Lösungsansätze, insbesondere dort, wo eine innovative, wirtschaftsnahe Kautelarpraxis wie die des Wagniskapitals zu bewerten ist. ${ }^{106}$ So kann der Funktionswandel von Geschäftsanteilen als Finanzierungsinstrument und Vergütungsbestandteil an der Peripherie des Unternehmens, mit starker Wirkung in den Markt adäquat abgebildet werden.

\section{Alternative Wirksamkeitsmaßstäbe}

Mehrere Alternativansätze scheinen vordergründig plausibel, sind allerdings dennoch zu verwerfen.

\section{a) Keine Prüfung am $A G B-R e c h t$}

Die im Markt verwendeten VC-Beteiligungsverträge wirken auf Grund der beschriebenen Rezeption angelsächsischer Standards durch einige erste Akteure in Deutschland homogen, wenn nicht sogar formularhaft, was eine Prüfung am AGB-Recht nahelegt. Richtig ist zwar, dass nicht schon deshalb eine AGBPrüfung entfällt, weil die Bereichsausnahme des $\$ 310$ Abs. 4 BGB für das Gesellschaftsrecht greift. ${ }^{107}$ Denn diese Vorschrift rechtfertigt sich vor allem aus

104 BGH NZG 2005, 968 verweist auf DNotZ 1991, 917, 918; ebenfalls pauschal STENZEL, $\mathrm{aaO}$ (Fn. 23), Rdn. 638; kritisch zur Vermengung von gesellschaftsrechtlichen und schuldrechtlichen Vereinbarungen, mit dem Fokus auf dem Schenkungsrecht BÜTTER/ Tonner, NZG 2003, 193, $194 \mathrm{ff}$ und BB 2005, 283, 285 ff; umfassend zum Fall HaberSACK/VERSE, ZGR 2005, 451.

105 „Mit sich ringend“, vgl. GeHrLeIn, NJW 2005, 1969, 1971.

106 So auch Kästle/Heuterkes, NZG 2005, 289, 291 f; zum Sonderproblem des AGGRechts, siehe Hornung, DB 2019, 1566. Zum rechtssoziologischen Ansatz grundlegend Luhmann, Rechtssystem und Rechtsdogmatik, 1974, S. 33 ff; DERs., Das Recht der Gesellschaft, 1993, S. 379ff; zur Legitimität ökonomischer und rechtsethischer Argumente in Auslegung und Rechtsanwendung Grundmann, RabelsZ 66 (1997), 423.

107 Offengelassen von BGH NZG 2005, 968, 971. 
der gesetzgeberischen Regelungsdichte im Gesellschaftsrecht. ${ }^{108}$ VC-Finanzierungen sind hingegen überwiegend Produkt der Kautelarpraxis, deren Muster die Berücksichtigung von Gemeinwohlbelangen nicht gleichermaßen sicherstellen und gerade an der Schnittstelle des Unternehmens zum Markt wirken, indem sie Investitionen sichern und Vergütung ersetzen. Wenn auch nicht die oben beschriebene Grundstruktur und Gesamtlogik der Beteiligungen zur Disposition steht, werden allerdings verschiedene Klauselalternativen, ihre konkrete Detailausgestaltung und die Gesamtkomposition des Beteiligungsvertrages sehr wohl individuell verhandelt. ${ }^{109}$ Wenn auch Beteiligungsmuster dominieren, sind Gründer und Mitarbeiter keine strukturell unterlegenen Parteien, sondern wie dargelegt Zentralgestalten im Startup und können selbst unter mehreren Musteralternativen auswählen und sie anpassen. ${ }^{110}$ Das betrifft etwa die Frage, welche der typischen Leaver-Fälle aufgenommen werden und welcher Abfindungsmodus gelten soll. Investoren wünschen oftmals sogar, dass Gründer selbst einen Beteiligungsvorschlag unterbreiten, da die Kontrolle gegenüber dem vollständigen Vertragsentwurf aus ihrer Perspektive kostengünstiger ist. ${ }^{111}$ Schließlich liegt bei Gründern und Mitarbeitern, die sich auf ein Vesting einlassen, ein hoher Bewusstseinsgrad über die Risiken des Geschäfts vor, was sie einer verbraucherähnlichen Schutzbedürftigkeit verlustig macht.

\section{b) Recht der Vertragsstrafe nicht einschlägig}

Auch eine Prüfung von Vesting-Regimen am Recht der Vertragsstrafe der $\int \mathbb{S} 339 \mathrm{ff}$ BGB scheidet aus. ${ }^{112}$ Denn eine Vertragsstrafe hat nach der dem BGB

108 Vgl. Basedow, Münchener Komm. z. BGB, 8. Aufl., 2019, \310 Rdn. 120.

109 Weitnauer, aaO (Fn. 1), Teil F Rdn. 109 ff; umfassend Kuntz, aaO (Fn. 1), 2. Teil, A., \$2, insbes. S. 272.

110 In diese Richtung etwa VentureCapital Magazin, 6/2017, 30f; zur Rechtfertigung des AGB-Rechts aus der Störung der Vertragsparität BASEDow, aaO (Fn. 108), Vor $\$ 305$ Rdn. 4-8; zur Unterscheidung zwischen AGB und „boilerplate contracts“ CHOI/GuLATI, Mich. L. Rev. 104 (2006) 1129, 1130; ferner zum Phänomen der Vermachtung im Privatrecht Möslein, Private Macht, 2016, dort insbesondere die Beiträge von MösLEIN, Private Macht als Forschungsgegenstand der Privatrechtswissenschaft, S. $1 \mathrm{ff}$ und Renner, Machtbegriffe zwischen Privatrecht und Gesellschaftstheorie, S. $505 \mathrm{ff}$.

111 Vom Gegenteil ausgehend wohl KunTz, aaO (Fn. 1), S. 272 ff.

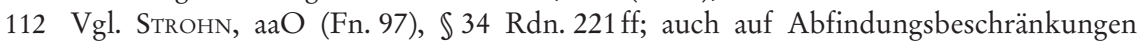
nicht anwenbar - dafür noch BGH WM 1983, 1207 (1208); Ulmer/Habersack, aaO (Fn. 93), Anh. $\ 34$ GmbHG Rdn. 104; a.A. BGH GmbHR 2014, 811 Rdn. $15 \mathrm{ff} \mathrm{m.}$ Anm. Wachter; Reuter, Verhandlungen des 55. DJT, 1984, Bd. I, B 66, S. 403 ff; SoufLeros, Ausschließung und Abfindung eines GmbH-Gesellschafters, 1983, S. 274f; allg. zu Verfallklauseln GotTwald, Münchener Komm. z. BGB, 8. Aufl., 2019, Vor \339 Rdn. 36 . 
zu Grunde liegenden Typologie einen Doppelcharakter - zum einen soll sie Präventionswirkungen zeitigen, ${ }^{113}$ zum anderen den Schadensersatz durch Pauschalierung erleichtern. ${ }^{114}$ Ausschluss und Abfindungsbeschränkung dienen zumindest im Fall der verschuldensabhängigen Bad-Leaver-Fälle auch dazu, den Gesellschafter zu disziplinieren. Denn anders als bei den bisher von der Rechtsprechung behandelten Mitarbeiterbeteiligungen, ${ }^{115}$ besteht neben dem Anteil oftmals keine regelmäßige, erhebliche Einkommensquelle aus dem Unternehmen, so dass eine empfindliche Vermögenseinbuße droht. Hingegen dürfte eine Pauschalierung des Schadensersatzes der Gesellschaft für das Fehlverhalten des Gesellschafters regelmäßig nur von ganz untergeordneter Bedeutung im Motivbündel des Vesting-Regimes sein. Für einen Schadensausgleich ist der Anteilsverlust auch zu undifferenziert, da der Schaden selbst nicht Tatbestand des Leaver-Falles ist. ${ }^{16}$ Wesentlicher ist der Belang der Leistungsbewertung des Gesellschafters im Leaver-Fall. Die Leistung, nicht das Verschulden, soll sich im Spiegel der dem Gesellschafter verbleibenden Anteile niederschlagen. Das ist die besondere Perspektive des „Erdienens" und entspricht dem gesetzgeberischen Leitgedanken von $\$ 614$ BGB. ${ }^{117}$ Damit fehlt ein Vertragsstrafencharakter. ${ }^{118}$

\section{c) Gesellschaftsrechtliche Grenzen der Vertragsfreiheit?}

Die immanenten Grenzen der Vertragsfreiheit waren für den BGH 1981 das tragende Argument für die Beschränkung des Rechts freier Hinauskündigung in einer Satzung. ${ }^{119}$ Der unbestimmte Begriff wurde als „Willkürformel“ und unter Bezugnahme auf die "Grundprinzipien des Gesellschaftsrechts" zur sog. „Damokles-Rechtsprechung" konkretisiert. ${ }^{120}$ Auch die Vertragsfreiheit rechtfertigt keinen bedingungslosen Gesellschafterausschluss. ${ }^{121}$ Allerdings geht es beim Vesting nicht um einen solchen. Zwar begibt sich der Gesellschafter se-

113 GotTwald, aaO (Fn. 112), $\$ 343$ BGB Rdn. 1

114 Vgl. BGH NJW 2003, 1805, 1808; NJW 2001,2622, 2624; NJW 2000, 2106, 2107; NJW 1995, 2788, 2790; NJW 1988, 2536; NJW 1983, 385, 387.

115 BGH WM 2014, vgl. den Überblick dazu bei GeHRLEIn, WM 2019, 1, 7.

116 Vgl. BGH WM 2014, 1343.

117 Vgl. dazu Stenzel, aaO (Fn. 23), Rdn. 610, 628.

118 Für den herkömmlichen Typus der Mitarbeiterbeteiligungen so auch GeHrLein, WM 2019, 1, 7.

119 BGH NJW 1981, 2565; unter Rekurs auf Wieland, Handelsrecht, Bd. I, 1921, S. 717; kritisch. Grunewald, aaO (Fn. 23), S. 128.

120 Vgl. etwa Huber, ZGR 1980, 177, 199. Kritisch zur Kohärenz der Kasuistik und den Bezügen zur Typenlehre, Grunewald, aaO (Fn. 23), S. $126 \mathrm{ff}$.

121 Flume, NJW 1979, 902 ff; so auch Huber, ZGR 1980, 177 (m. w. N. in Fn. 72); vgl. zum aktuellen Stand des Arguments, zwischen Rechtsprinzip und „mystifizierendem Leuchtfener“, RIESENHUBER, ZfPW 2018, 352. 
henden Auges in eine Abhängigkeitssituation. ${ }^{122}$ Diese ist indes nicht absolut und dient einem ökonomischen Kalkül. Die „umgekehrte Anwartschaft“ im Reverse-Vesting realisiert das gewünschte Geschäft „Arbeit gegen Beteiligung und Expektanz" oder "Kredit gegen Beteiligung und Expektanz". Wie dargelegt sind die Risiken für die Parteien evident. Zudem sind Vesting-Regime kein Produkt einseitigen Diktats, sondern professioneller Interessenvertretung. ${ }^{123}$ Der Gesellschafter gibt dabei nicht die Kontrolle über den Ausschlussgrund auf, sondern setzt diesen lediglich niedrigschwellig an, wie etwa im Fall des Ausschlusses wegen Verfehlung von Zielen aus dem mit den Investoren festgelegten Geschäftsplan, um im Gegenzug von Wertsteigerungen seiner Anteile profitieren zu können. Sein Status ähnelt dem des anerkannten „Gesellschafters minderen Rechts". ${ }^{124}$ Dies ist bei der Bewertung der jeweiligen Klauseln zu berücksichtigen. ${ }^{125}$ Stat pro ratione voluntas gilt auch im Zusammenhang mit einer bewussten Selbstgefährdung und Risikogeschäften im Gesellschaftsrecht. ${ }^{126}$ Optimismus und ggf. selbstverschuldet schlechte Beratung machen nicht automatisch schutzwürdig. ${ }^{127}$ Die Kontrolle der Grenzen der Vertragsfreiheit sollte nur ihre extremen Grenzen erfassen, da diese anderenfalls der richterlichen Bevormundung der Parteien zum Opfer fiele. Zweifel an der wirtschaftlichen Sinnhaftigkeit sollten nicht hinreichen.

\section{d) Treu und Glaube sowie gesellschaftsrechtliche Trenepflicht}

Treu und Glaube sowie die noch weitere gesellschaftsrechtliche Treuepflicht erlauben die Betrachtung eines individuellen, konkreten Parteiverhaltens unter Bezugnahme der Parteihorizonte, insbesondere des Gesellschaftszwecks und der „Realstruktur“ der Gesellschaft. ${ }^{128}$ Dieser Maßstab gilt gegenüber der Sit-

122 Ganz im Gegensatz zum legendären Damokles, der aus Genusssucht einen ihm nicht gebührenden Platz an der Herrschertafel sucht und sich in der Falle, unter einem an Rosshaar gebundenen Schwert wiederfindet, Cicero, tusculanae disputationes 5, 6162; die Metapher wurde in das Gesellschaftsrecht eingeführt durch SchilLing, ZGR 1979, 419, 426; kritisch dazu etwa auch Flume, DB 1986, 629.

123 Siehe dazu sogleich IV. 1.

124 Dazu Flume, NJW 1979, 902; Ders., Allgemeiner Teil des Bürgerlichen Rechts, Bd. I/1, 1977, \10 III; vgl. auch Huber, ZGR 1980, 177, $191 \mathrm{ff}$.

125 Zum Erfordernis der Gesamtwürdigung bei der Beurteilung der Sittenwidrigkeit in Gesellschaftsverhältnissen, vgl. BGH NZG 2013, 985 Rdn. 29; Armbrüster, aaO (Fn. 97), $\ 138$ BGB Rdn. 80.

126 Vgl. dazu Fuume, Allgemeiner Teil des Bürgerlichen Rechts, Bd. II., Das Rechtsgeschäft, 4. Aufl., 1992, \$1 5, S. 6; vgl. Göz/Kowalewski, NZG 2018, 1369, 1375 - Verweis auf BGH NZG 2012, 259, 261.

127 So auch Huber, ZGR 1980, 211.

128 Vgl. Merkt, Münchener Komm. z. GmbHG, 3. Aufl., 2018, $\mathbb{1 3}$ Rdn. 88ff, 95; FASTRICH, in: Baumbach/Hueck, Komm. z. GmbHG, 22. Aufl., 2019, \13 Rdn. 22. 
tenwidrigkeit als strenger, da die Sittenwidrigkeit eine Bewertung allein anhand einer abstrakten sozialen Normierung ermöglicht. ${ }^{129}$ Die kapitalistische Ausgestaltung einer Gesellschaft reduziert allerdings die Treupflicht. ${ }^{130}$ Vor allem ist sie umso weiter reduziert, je konkreter die dem Verhalten zu Grunde liegende Vereinbarung ist. Denn dann ist gerade nicht das bei Langzeitverträgen grundsätzlich erforderliche Ergänzungs- und Präzisierungsinteresse betroffen. ${ }^{131}$ Bei genau ausdifferenzierten Vesting-Klauseln sind daher die Parteien zumindest unter dem Topos von Treu und Glauben weniger schutzbedürftig. ${ }^{132}$ Allerdings bleibt für den Treuegedanken Raum bei der Ausübung des in dem Vesting-Regime festgelegten Ausschlussrechts (dazu noch unten).

\section{Wirksamkeitsvermutung bei marktüblichen Klauseln}

\section{a) Sittenwidrigkeitskontrolle durch Interessenabwägung}

Da keines der geprüften Alternativkonzepte hinreichend überzeugend ist, muss auch für Produkte der VC-Kautelarpraxis an der Hinauskündigungsrechtsprechung festgehalten werden. Sie kann als solche allerdings Ausgangspunkt für wirtschaftssoziologische Erwägungen sein, da ihr dogmatischer Sockel die Sittenwidrigkeit ist. Hier sind mit Teubner zunächst die historische Kontingenz und der Bedeutungswandel der Norm hervorzuheben. ${ }^{133}$ Der Definition guter Sitten durch das RG als „Anstandsgefübl aller billig und gerecht Denkenden" ist kein großes Gewicht mehr beizumessen. ${ }^{134} \$ 138$ BGB stellt inzwischen unzweifelhaft lediglich einen rechtsethischen Mindeststandard mit geringer Prüfungsdichte dar. ${ }^{135}$ So sind für die Sittenwidrigkeitskontrolle ka-

129 So etwa Grunewald, aaO (Fn 23), S. $132 \mathrm{ff}$; BGHZ 64, 238, $241 \mathrm{ff}$; allerdings ist auch die mitgliedschaftliche Treuepflicht anerkannt, МеRкт, aаO (Fn. 128), $₫ 13$ GmbHG Rdn. 98. Zur geminderten Bedeutung des Wortlautes und Tatbestandsmerkmals „mit Rücksicht auf die Verkehrssitte“, SCHUBERT, Münchener Komm. z. BGB, 8. Aufl., 2019, $\$ 242 \mathrm{Rdn} .2$.

130 Fastrich, aaO (Fn. 128), \13 GmbHG Rdn. 22.

131 Fastrich, aaO (Fn. 128), $\$ 13$ GmbHG Rdn. 20; umfassend dazu die Beiträge in NicKLISCH, Der komplexe Langzeitvertrag, 1987.

132 Kritisch zur Schutzbedürftigkeit von Kapitalgebern im Gesellschaftsrecht allgemein, m.w. N. Grunewald, aaO (Fn. 23), S. 134 f.

133 Dazu Teubner, aaO (Fn. 79), S. $65 \mathrm{ff}, 115 \mathrm{ff}$.

134 \727 Mot. II; BGHZ 179, 218, stRspr seit RGZ 48, 124; „trägt zur inhaltlichen Präzis ierung des $\ 138$ wenig bei“: Ellenberger, in: Palandt, Komm. z. BGB, 80. Aufl., 2021, \138 Rdn. 2; zurückhaltender Armbrüster, aaO (Fn. 97), $\$ 138$ BGB Rdn. 14 f.

135 Siehe bei Grunewald, aaO (Fn. 23), S. 128. Zur Unbestimmtheit des Begriffs etwa EllenBERgER, aaO (Fn. 134), $\$ 138$ BGB Rdn. 2; zur Prüfung von Satzungsbestimmungen über die Einziehung am Maßstab des $\$ 138$ BGB, vgl. Strohn, aaO (Fn. 97), $\$ 34$ Rdn. 57f; zur Prüfung von Nebenabreden am Maßstab von $\$ 138$ BGB, BGH II ZR 173/04. 
suistisch anerkannte Elemente prägend, die, obschon breit gestreut, im Verhältnis der Wechselwirkung zueinander stehen. ${ }^{136}$ In gesellschaftsrechtlichen Zusammenhängen stehen dabei Freiheitsbeschränkungen und Machtmissbrauch im Vordergrund. ${ }^{137}$ Praktikabel gemacht wird die Norm letztendlich implizit in Folge einer Delegationstheorie, bei welcher der Richter das Parlament ersetzt - durch eine quasi-gesetzgeberische Interessenabwägung. ${ }^{138}$ Das Rechtsgeschäft ist umfassend zu erfassen, von Inhalt, Motiv und Zweck bis hin zu Zustandekommen, Umsetzung und Rechtsfolge. ${ }^{139}$ Diese Maßgaben bleiben freilich weit geschnitten und so wirft die Interessenabwägung seit jeher und über die Hinauskündigungsdogmatik hinaus erhebliche methodische Probleme auf. ${ }^{140}$ Die Abwägung birgt im Vergleich zu genau ausformulierten Tatbeständen und Rechtsbegriffen offenkundig ein höheres Maß an Rechtsunsicherheit. Sie hat freilich sukzessive eine strenge Begriffsdogmatik zumindest ergänzt. ${ }^{141}$ Tatbestand und Rechtssatz - abstrakter gesprochen: Faktizität und Normativität, Normanwendung und Norm - werden inzwischen bereitwillig verschliffen. Genaues Sachverhaltsverständnis füllt die Freiräume unbestimmter Rechtsnormen aus. ${ }^{142}$ Dieser allgemeine Verschleifungsprozess von Nor-

136 Sittenwidrigkeitskriterien als „bewegliche Elemente“, vgl. Armbrüster, aaO (Fn. 97), $\$ 138$ BGB Rdn. 27 ff; zum Grundgedanken eines beweglichen Systems, WiLBURG, Entwicklung eines beweglichen Systems im bürgerlichen Recht, 1951; DERS., AcP 163 (1964), $346 \mathrm{ff}$; vgl. dazu Canaris, Systemdenken und Systembegriff in der Jurisprudenz, 1969, S. 74ff; Westerhoff, Die Elemente des Beweglichen Systems, 1991; zur Konkretisierung von Generalklauseln durch das bewegliche System Byduinski, in: Behrends u.a., Rechtsdogmatik und praktische Vernunft, 1990, S. $189 \mathrm{ff}$; kritisch zur herausgebildeten Kasuistik und den historischen Funktionswandel der Norm betonend Teubner, aaO (Fn. 79), S. $115 \mathrm{ff}$.

137 Vgl. zu den Fallklassen Armbrüster, aaO (Fn. 97), \$138 BGB Rdn. 27 ff, 33 ff; zur Anwendung auf Management-Beteiligungen SchockenhofF, ZIP 2005, 1009, 1015.

138 Grundlegend Arzt, Die Ansicht aller billig und gerecht Denkenden, 1965, S. 99; KrafT, Interessenabwägung und Gute Sitten im Wettbewerb, 1963, S. 42.; m.w. N. Teubner, aaO (Fn. 79), S. $42 \mathrm{ff}, 106 \mathrm{ff}$.

139 Vgl. nur Ellenberger, aaO (Fn. 134), $\$ 138$ BGB Rdn. 8.

140 Dies war etwa schon in der Wertungsjurisprudenz stark umstritten, siehe bei LARENZ, Methodenlehre der Rechtswissenschaft, 6. Aufl., 1991, Kapitel 5, S. $119 \mathrm{ff} ;$ zu den verschiedenen im Gesellschaftsrecht zu berücksichtigenden Interessen im zeitlichen Wandel vgl. etwa m.w. N. Wiedemann, ZGR 1980, 147, $149 \mathrm{f}$.

141 BVerfG BVerfGE 7, 198, 210 - Lüth; dazu LadeUr, RTh 2014, 467, 480 ff; zum Kollisionsverhalten von Rechtsprinzipien vgl. Canaris, aaO (Fn. 136), S. 53 ff, Bydlinski, AcP 204 (2004), 309; siehe auch RufFerT, Vorrang der Verfassung und Eigenständigkeit des Privatrechts, 2001, S. $99 \mathrm{ff}$ sowie Tischiırek, Die Verhältnismäßigkeitsprüfung Methodenmigration zwischen öffentlichem Recht und Privatrecht, 2017, insbes. S. $137 \mathrm{ff}$; zum Methodendiskurs im Gesellschaftsrecht zuletzt RissE/HöfLiNG, NZG 2017, 1131.

142 Dazu früh etwa - Kantorowicz, Der Kampf um das Recht, 1909; vgl. weiter bei LARENZ, aaO (Fn. 140), S. $128 \mathrm{ff}$ und allgemein S. $262 \mathrm{ff}$. 
men ist durch die Bildung von Spezialwissen in immer komplexeren und durch Organisationen außerhalb des Staates geprägten Gesellschaften verstärkt, die ein in gesetzlichen Normbegriffen kodifizierbares „Allgemeinwissen“ fast ganz ausschließen. ${ }^{143}$ Die vom Gesetzgeber weitestgehend unbegleitete Herausbildung eines neuen Feldes der Kautelarjurisprudenz im Venture Capital ist dafür ein mustergültiges Beispiel.

\section{b) Gebot eines wirtschaftssoziologischen Ansatzes}

Aus ökonomischer und freiheitlicher Perspektive liegt es nahe, die Gestaltungräume der Marktpraxis zu bewahren und allenfalls punktuell besondere gesetzgeberische Regelungen einzuführen. Dies dürfte auch im Geiste eines soziologisch aufgeklärten Rechtswesens liegen, das sich als Teil des Gesamtgesellschaftssystems versteht. Die damit mittelbar verbundene Konfliktlösung über die Interessenabwägung kollidiert formal mit rechtlichen Kerngewährleistungen, insbesondere der Vorhersehbarkeit und Gleichheit. ${ }^{144}$ Freilich ist spezialisierten, risikoaffinen und an Komplexität gewöhnten Marktteilnehmern, zumal bei der üblichen intensiven anwaltlichen Beratung, ein gewisses Maß an Unsicherheit zumutbar. ${ }^{145}$ Eine verständige Würdigung der tatsächlichen wirtschaftlichen, ideellen und sozialen Verhältnisse und Motive der Parteien, um im besonders gelagerten Einzelfall eine sittenwidrige Regelung zu vermeiden, ist dafür Voraussetzung. So kann dann zu Recht die Rede von einer Rezeptionsfunktion der Sittenwidrigkeitskontrolle sein, die gerade dort außerrechtlichen Wertungen Geltung verschaffen soll, wo die Verkehrskreise keiner paternalistisch festzustellenden öffentlichen Ordnung bedürfen. ${ }^{146}$ Eine wesentliche Berücksichtigung der Marktpraxis bei der Wirksamkeitskontrolle entspricht dem durch Max Weber vorgeprägten Konzept „soziologischer Aufklärung des Rechts " ${ }^{147}$ und legitimiert sich institutionenökonomisch, insbesondere nach der von bayekschen Informationstheorie vor allem durch den Ge-

143 Grundlegend dafür HaYeK, Die Anmaßung von Wissen, 1974.

144 Kritisch und mit weiteren Erwägungen Luhmann, aaO (Fn. 106), S. 279, 318, 528, 539.

145 Zur - neuerlichen - Verdrängung einer Begriffsdogmatik durch Abwägung LADEUR, RTh 2014, 467; sowie Ders., Archiv für Rechts- und Sozialphilosophie, Vol. 69, No. 4 (1983), pp. 463-483.

146 Teubner, aaO (Fn. 79), S. 65 ff; weiterführend Bydlinski, aaO (Fn. 136), S. 189, $198 \mathrm{ff}$.

147 M. Weber, Rechtssoziologie. Neuwied, 1960, S. 334; zur zentralen Methode der Typenbildung in Webers Rechtssoziologie Petersen, Max Webers Rechtssoziologie und die juristische Methodenlehre, 3. Aufl., 2020, S. 110 ff; vgl. mit diesem Ansatz bei der AGB-Kontrolle Renner, AcP 213 (2013), 677, 691 f, 695, mit Verweis auf HAYeK, Ordo 26 (1975), 12; Luhmann, Soziologische Aufklärung, 1969; für eine responsive Rechtswissenschaft Grünberger, AcP 218 (2018), 213 ff; siehe schon den Ansatz von Esser, die „Guten Sitten“ als Standards zu begreifen, Grundsatz und Norm, S. $96 \mathrm{ff}$ sowie den Überblick von Teubner, aaO (Fn. 79), S. 45 ff. 
danken, „besserem“ Wissen im dezentralen Wirtschaftsgeschehen zur Geltung zu verhelfen. ${ }^{148}$ Die erheblichen Kostenvorteile durch Spezialisierung auf ein „Men̈̈ von Musterklauseln“ sind ebenfalls von Gewicht. ${ }^{149}$ Ein neukantianischer Einwand, „Sollen“ dürfte nicht vom „Sein“ beeinflusst werden, vermag angesichts der Vorteile und Limitierung privater Regelsetzung nicht zu verfangen - und kann die Exklusivität der Delegation der Regelsetzungsbefugnis an Richter statt an - und gegen! - Teile der Gesellschaft nicht zufriedenstellend begründen. ${ }^{150}$

Als Synthese des Vorgesagten sollte im Sinne eines ,judicial self-restraint “, also der richterlichen Zurückhaltung, ${ }^{151}$ die Prüfungsintensität bei im Markt zwischen professionellen Parteien etablierten Standards zurückgenommen werden. ${ }^{152}$ Die darin geronnenen Handelsbräuche müssen auch im Rahmen der Sittenkontrolle des $\ 138$ BGB relevant sein - denn Handelssitten sind wegen ihres normativen Ursprungs nicht sittenwidrig. ${ }^{153}$ Auch eine Sanktionierung von in Handelsbräuchen kondensierten „Unsitten“, also von allen billig und gerecht denkenden Außenseitern abzulehnenden Regelungen, sollte nicht über $\$ 138$ BGB erfolgen, wenn der normative Geltungsgrund von Handelsbräuchen anerkannt und die Rezeptionsfunktion der Sittenwidrigkeitskontrolle bewahrt werden soll. Erforderlich ist dafür vielmehr eine ausdrückliche gesetz-

148 Hayek, The Use of Knowledge in Society, The American Economic Review, Vol. 35, No. 4 Sept. 1945, 519; DERs., aaO (Fn. 143); zum Wissen in vernetzten Gesellschaften auch Castells, The Rise of the Network Society, 1996, S. $92 \mathrm{ff}$. Kritisch zur Rechtfertigung der Selbstregulierung durch Transaktionskosten, mangels Quantifizierbarkeit Merkt, aaO (Fn. 4), S. 167, 171.

149 Dazu Bengtsson/Bernhardt, Journal of economics \& management strategy, 2014-06, Vol.23 (2), p.396, 401.

150 Siehe m.w.N. Teubner, aaO (Fn. 79), S. 36; Luhmann, aaO (Fn. 106), S. 146f; siehe auch Denga/Pohle/Hölzel, RW 11(2020), 418, 423f; zur historischen DelegationsTheorie Kraft, aaO (Fn. 138), S. 42; erste spezifische Anlagen schon in den $\$ \mathbb{S} 24 \mathrm{ff}$ GWB, in Form der Wettbewerbsregeln, die freilich in einem zentralisierten Prozess durch Wirtschafts- und Berufsvereinigungen aufgestellt werden können, der mit zu ihrem praktischen Bedeutungsverlust geführt haben dürfte, dazu BGH v. 7.2.2006, WuW/E 1779, 1782 „Probeabonnement“; krit. hierzu Alexander, ZWeR 2007, 239, 250. Zur induktiven Methode der Rechtswissenschaft Metzger, aaO (Fn. 81), S. $47 \mathrm{ff.}$

151 Grundlegend zu diesem Konzept Cover, Harvard Law Review 97 (1983), 4.

152 Vgl. auch zu einer "Theory of Boilerplate Interpretation“, welche die Prüfungskompetenz primär Fachgremien des Marktes zuweisen will CHOI/Gulati, 104 Mich. L. Rev. 1129 (2006), 1159 ff; zu diesem Ansatz schon Teubner, aaO (Fn. 79), S. 31 ff, 92 ff; noch weitergehend RENNER, AcP 213 (2013), 677, 715.

153 Sollte $\mathbb{} 242$ BGB als Ansatzpunkt der Kontrolle gewählt werden, so müsste in der Norm das Merkmal „mit Rücksicht auf die Verkehrssitte“ ernst genommen werden die h.M. beklagt etwa zu wenig empirische Untersuchungen der Verkehrssitte und die Ausfüllung des Merkmals durch richterliche Wertungen, etwa SCHUberT, aaO (Fn. 129), $\$ 242$ BGB Rdn. 13; mit Beispielen für „Missbrauch“ Hopt, in: Baumbach/ Hopt, Komm. z. HGB, 40. Aufl., 2021, $\$ 346$ Rdn. 11. 
geberische Wertung mit zivilrechtlicher Sanktionierung über $\$ 134$ BGB, was das Legitimationsproblem richtigerweise in die Ebene des öffentlichen und Verfassungsrechts mit seinen diskursiven Absicherungen verlagert. ${ }^{154}$

Ergeben sich aus dem Einzelfall also keine Hinweise auf eine grob unpassende Ausgestaltung von Musterklauseln, ist materiell-inhaltlich von der Wirksamkeit der Ausschlussklausel auszugehen. Wie oben gezeigt spiegeln VestingKlauseln eine rationale Motivation der Parteien wider, die gewiss von spekulativen Expektanzen durchsetzt ist, allerdings letztendlich ausgewogene Antworten auf das Principal-Agent-Dilemma gibt. Motivationswirkung und Leistungsanreiz, ${ }^{155}$ Sicherung der Mitarbeit, ${ }^{156}$ und Test der Zusammenarbeit ${ }^{157}$ sind immerhin auch vom BGH anerkannte Interessenlagen beim Gesellschafterausschluss. VC-Musterklauseln indizieren somit im Kontext der Interessenabwägung ein Interessengleichgewicht. Bei der Wirksamkeitsbewertung marktüblicher VC-Beteiligungen sind daher Inhalte, Motive und Zwecke der Parteien im Einzelfall grundsätzlich nicht mehr zu prüfen. Darlegungs- und Beweislast für eine marktunübliche Ausgestaltung der Musterklauseln obliegen demjenigen, der sich auf sie beruft.

Ohne einen solchen materiell-rechtlichen Ansatz droht der ohnehin erhebliche Abfluss von Schlichtungsfragen an Schiedsgerichte sich noch weiter zu Lasten staatlicher Gerichte zu verstärken. ${ }^{158}$ Staatliche Gerichte müssen sich gerade bei Anwendung der Generalklauseln offen gegenüber dem „selbstgeschaffenen Recht der Wirtschaft" zeigen. ${ }^{159}$ Erkennen sie dessen erprobte Lösungen nicht an, so kann dies einen Verlust für die Attraktivität deutschen Rechts an sich bedeuten. Im hier vertretenen Zusammenspiel von Kautelarpraxis und Gerichtswesen wäre eine „bybride Selbstregulierung "160 verwirklicht, der auch kein Marktversagen in Form negativer externer Effekte der VC-Beteiligungspraxis entgegensteht. Denn nur solche können aus ordoliberaler Sicht Regulierung - und damit auch das Eingreifen von Kontrollnormen zwingenden Privatrechts - rechtfertigen. ${ }^{161}$ Auch ordnungspolitische Ziele zum Schutz

154 Dazu konzeptionell grundlegend Habermas, Faktizität und Geltung, 1992, insbes. S. $349 \mathrm{ff}$.

$155 \mathrm{Zu}$ diesen Aspekten bei Management-Beteiligungen BGH NJW 2005, 3641; NJW 2005, 3644; Habersack/Verse, ZGR 2005, 451, $461 \mathrm{ff}$.

156 BGH NJW 1983, 2880, 2881.

157 BGH NJW 2004, 2013, 2015.

158 Dazu etwa G. Wagner, Rechtsstandort Deutschland im Wettbewerb, 2018, S. $91 \mathrm{ff}$.

159 So schon früh, RüHL, aaO (Fn. 5), S. 16.

160 Merkt, aaO (Fn. 4), S. 167, 169.

161 Siehe etwa Ruffert, in: Fehling/Ruffert, Regulierungsrecht, 2010, $\mathbb{7}$ Rdn. 19; EIfERT, Regulierungsstrategien, in: Hoffman-Riem/Schmidt-Aßmann/Voßkuhle, GVerwR I, 2. Aufl., 2012, $\ 19$ Rdn. 16ff; BASEDOw, FS Immenga, 2004, S. 3, 8; zur ökonomischen Theorie von Externalitäten grundlegend Buchanan/Stubblebine, Extemality, in: Eco- 
strukturell unterlegener Parteien sind nicht ersichtlich, da es sich gerade nicht um eine asymmetrische Situation handelt, sondern um einen hochprofessionellen Kontext von Waffengleichheit zwischen Investoren, Gründern und Mitarbeitern.

\section{Neuralgische Regelungsthemen bei Wagniskapitalbeteiligungen}

Geht man davon aus, dass Musterklauseln grundsätzlich ein Interessengleichgewicht indizieren, ist es für die rechtssichere Ausgestaltung der Vesting-Verträge wesentlich, diejenigen Regelungsthemen zu erfassen, die neuralgisch für fehlerhafte Ausgestaltungen und Störungen des Interessengleichgewichts sind. Bei ihnen sind Marktüblichkeit und abweichende Alternativgestaltungen besonders sorgfältig zu prüfen. Auch hier fördert Typenbildung Rechtssicherheit schon bei der Vertragsgestaltung. ${ }^{162}$

\section{a) Reichreite des Anteilsverlusts}

Je weiter der Anteilsverlust durch Vesting-Regime reicht, desto eher können die beiden Sittenwidrigkeitsklassen von Machtmissbrauch und freiheitsbeschränkender Verhaltenssteuerung vorliegen. Bad-Leaver-Klauseln können ein Interessenungleichgewicht eher hervorrufen, als Good-Leaver-Klauseln, da bei letzteren dem Gesellschafter immer noch die erdienten Anteile verbleiben. Allerdings können auch Good-Leaver-Klauseln, die den vollständigen Anteilsverlust nicht ausdrücklich vorsehen, in Verbindung mit dem Zeitelement alle Anteile betreffen, wenn die Cliff-Periode noch nicht überschritten ist. Bei besonders späten Cliff-Zeitpunkten ist ein Interessenungleichgewicht nicht mehr auszuschließen. Relevant ist auch, wie steil die Vesting-Kurve verläuft, also wie viele Anteile pro Zeiteinheit fest erdient werden, und schließlich auch, ob auch diejenigen Anteile verloren gehen, die der Gesellschafter vor der Verabredung des Vesting-Regimes innehatte und wie gegebenenfalls die verbleibenden Anteile beschränkt werden. ${ }^{163}$

nomica 29 (1962), S. 371 ff, S. 372; siehe auch Bachmann, aaO (Fn. 3), S. 54 f; für eine Berücksichtigung lediglich bei asymmetrischen Verträgen HabERSACK, Vertragsfreiheit und Drittinteressen, 1992, insbes. S. $160 \mathrm{ff}$.

162 Zur Typisierung im Recht vgl. Larenz, aaO (Fn. 140), S. 461 ff, sowie zur Operationalisierung eines „beweglichen Systems“ von Tatbestandselementen S. 478.

163 Vgl. dazu Bank/Möllmann, aaO (Fn. 23), Teil 3 Rdn. 239ff; Samios/Arnold, aaO (Fn. 23), S. 151, 153. 


\section{b) Kontrolle der Leaver-Tatbestandsverwirklichung}

Die Vorhersehbarkeit der Tatbestandsverwirklichung entwickelt Grunewald als zentrales Kriterium für die Zulässigkeit von satzungsmäßigen Ausschlussklauseln. ${ }^{164}$ Dieser Ansatz ist auch bei VC-Beteiligungen wichtig, so dass hierauf ein besonderes Augenmerk bei der Kontrolle der Marktüblichkeit liegen muss. Für die Frage der Wirksamkeit von Ausschlussklauseln ist besonders relevant, inwieweit der Gesellschafter zu zumutbaren Bedingungen die Tatbestandsverwirklichung des Leaver-Falles kontrollieren und verhindern kann. Ein willkürlicher Tatbestand, der außerhalb des tatsächlichen Einflussbereichs des Gesellschafters liegt, kann in sich schon machtmissbräuchlich sein und die Entscheidungsfreiheit des Gesellschafters zu stark beschränken. Hingegen sind Ausschlussgründe, die an das Verhalten oder Verschulden des Gesellschafters anknüpfen, ${ }^{165}$ Indiz für die Wirksamkeit der Klausel. ${ }^{166}$ Kritisch sind Ausschlussklauseln, die auf die ordentliche Kündigung eines Dienstverhältnisses durch die Gesellschaft abstellen. Werden Gesellschafterstellung und Dienstverhältnis auf diese Weise verknüpft, kann die Gesellschaft mittelbar über den Anstellungsvertrag auch den Gesellschaftsstatus kontrollieren. ${ }^{167}$

Bei normativ-offen formulierten Klauseln kann auch das Verfahren zur Tatbestandsfeststellung mit in die Prüfung einfliessen. Die Feststellung durch ein Gremium, etwa die Gesellschafterversammlung, bietet grundsätzlich eine höhere Richtigkeits- und Fairnessgewähr als die Entscheidung Einzelner. ${ }^{168} \mathrm{Al}-$ lerdings können auch dort Einzelakteure dominieren, weshalb die formelle Tatbestandsfeststellung insgesamt eher ein untergeordnetes Kriterium für die Wirksamkeit der Klausel bleiben dürfte.

\section{c) Umfang der Abfindung}

Die Abfindungsregelung gilt als problematischer Anhang von Ausschlussklauseln und wird - wenn nicht die Kapitalerhaltung in Frage steht - getrennt von ihnen behandelt. ${ }^{169}$ Jedenfalls in Vesting-Regimen ist der Anteilsverlust jedoch

164 Grunewald, aaO (Fn. 23), S. 193 ff; maßgeblich unter Bezug auf BGH NJW 1989, 834, 835 f; zum Transparenzgedanken als „Strukturprinzip“ privater Regelsetzung, siehe Bachmann, aaO (Fn. 3), S. 328.

165 Auf das Verschulden abstellend Weitnauer, aaO (Fn. 1), Teil F. Rdn. 220.

$166 \mathrm{Zu}$ den beim Gesellschafterausschluss herkömmlich anerkannten Fallgruppen, vgl. schon oben V. 1 .

167 Habersack/Verse, ZGR 2005, 451, 455.

168 In diese Richtung, BGH NZG 2005, 479, 480; Habersack/Verse, ZGR 2005, 451, 457; Grunewald, aaO (Fn. 23), S. 131.

169 Vgl. Huber, ZGR 1980, 177, 184; Gerhlein, NJW 2005, 1969; Ders. WM 2019, 1, 6 ff; zu dem Ganzen auch Habersack/Verse, ZGR 2005, 451, $472 \mathrm{ff}$. 
untrennbar mit der Entschädigungsfrage verwoben, da der Anteilsverlust differenziert und gemeinsam mit der Abfindung geregelt wird. ${ }^{170}$ Allerdings muss auch bei der Einbeziehung des Abfindungsumfangs der sozio-ökonomische Kontext berücksichtigt werden. Huber lehnt eine Interessenabwägung bei der Frage des Abfindungsausschlusses und eine Nachverhandlung der Vergütung „wirtschaftlicher Fübrungskräfte“ als „einzigartiges und durch nichts zu rechtfertigendes Privileg!" gar vollständig ab. ${ }^{171}$ Bei den Vertragsparteien von Vesting-Regimen handelt es sich zwar nicht allein um Manager, sondern auch um Arbeitnehmer. Indes sind die unter Mitarbeiterbeteiligungsprogrammen mit echten Geschäftsanteilen bedachten Arbeitnehmer keine schwachen Figuren in wirtschaftlichen Verhandlungen. ${ }^{172}$ Fachkräfte können inzwischen häufig wie Manager ihre Interessen durchsetzen. Diese Rollenrationalität im hochprofessionellen Kontext der VC-Beteiligungen spricht daher auch bei fehlender Marktüblichkeit der Beteiligungsklausel gegen eine tragende Rolle des Abfindungsumfangs bei der Frage nach einer Klauselwirksamkeit.

\section{d) Verfügbare Rechtsbehelfe}

Rechtsbehelfe wie etwa Anfechtungsklage oder Widerspruch gegen die Änderung der Gesellschafterlisten beim Notar, spielen eine eher untergeordnete Rolle bei der Wahrung des Interessengleichgewichts der Parteien. Denn hier muss die behavioristische Erkenntnis berücksichtigt werden, dass Trägheitseffekte und die Abschreckungswirkung des allgemeinen Prozessrisikos von ihrer Wahrnehmung abhalten. ${ }^{173}$ Die Einbeziehung von Rechtsbehelfen in die materiell-rechtliche Bewertung von Rechtsgeschäften wäre zirkelschlüssig und ist daher eher kein geeigneter Ansatzpunkt für die Überprüfung der Marktkonformität einzelner Klauseln.

170 So auch der Ansatz von Grunewald, aaO (Fn. 23), S. 9 ff und die Rechtsprechungsnachweise auf S. 12 (Fn. 12); siehe auch den globaleren Ansatz das Gebot von Verhältnismäßigkeit mit dem Verbot von Unverhältnismäßigkeit zu koppeln Hanau, in: Möslein, Private Macht, 2016, S. $119 \mathrm{ff}$ sowie DERs., Der Grundsatz der Verhältnismäßigkeit als Schranke privater Gestaltungsmacht, 2004.

171 Vgl. Huber, ZGR 1980, 177, 195.

172 Für breiter aufgesetzte Mitarbeiterbeteiligungsprogramme, die sich auch an weniger verhandlungsstarke Arbeitnehmer richten, wie insbesondere VSOPs könnten Aufklärungs- und Beratungspflichten analog der $\mathbb{S} 63 \mathrm{ff}$ WpHG erwogen werden.

173 Zum Problem der Trägheitseffekte Hacker, Verhaltensökonomik und Normativität, 2017, S. $488 \mathrm{ff}$. 


\section{Umstandskontrolle}

Die Sittenwidrigkeitskontrolle nach $₫ 138$ BGB bezieht sich ebenfalls auf die Umstände des Rechtsgeschäfts. ${ }^{174}$ Das Umstandsmoment ist nicht von der hier vertretenen Wirksamkeitsvermutung erfasst, da die VC-Muster allein auf $m a-$ teriell-inhaltlicher Ebene die Marktpraxis abbilden.

Möglich und erforderlich bleibt also die Überprüfung der Umstände des $\mathrm{Zu}$ standekommens der VC-Beteiligung unter dem Gesichtspunkt der Sittenwidrigkeit. ${ }^{175}$ Dabei ist die Zustimmung zum Rechtsgeschäft lediglich notwendiges Legitimitätskriterium. Daneben sind alle Umstände der Einführung eines Vesting-Regimes mit zu berücksichtigen. Zu hinterfragen ist der tatsächlich eröffnete Spielraum, denn die Vertragsfreiheit kann im Einzelfall faktisch auf eine bloße Vertragsabschlussfreiheit verkürzt sein und die gestalterischen Elemente des Vertragskompromisses einbüßen. ${ }^{176}$ Andererseits ist das „Versagen gegen sich selbst", eigene Interessen trotz bestehender Möglichkeit nicht adäquat in Vertragsverhandlungen durchzusetzen, gerade im Unternehmens- und Investitionsverkehr beachtlich. ${ }^{177}$ Denn ansonsten wären sorgfältige, abwägende und Beratung suchende Verkehrsteilnehmer benachteiligt. ${ }^{178}$

Die Beteiligung an Neugründungen unterscheidet sich fundamental vom Eintritt in bestehende Strukturen. ${ }^{179}$ Strukturell können beim Beitritt in einen bestehenden Verband Partikularinteressen schlechter durchgesetzt werden, da der Beitrittsinteressent über von der bestehenden Gesellschaftermehrheit geschaffene Fakten verhandeln muss. Komplexe Gruppenprozesse unter dem Mehrheitsprinzip wirken dabei hinderlich auf die freie unternehmerische Entfaltung des Gesellschafters. ${ }^{180}$ Berücksichtigungsfähig ist freilich auch der Um-

174 Vgl. etwa Habersack/Verse, ZGR 2005, 451, 471 f; hier den Schwerpunkt setzend VerSE DStR 2007, 1822 ff; allgemein auch Ellenberger, aaO (Fn. 134), \138 BGB Rdn. 8.

175 BGH VII ZR 132/97; Dörner, in: Schulze, Bürgerliches Gesetzbuch, 10. Aufl., 2019, $\$ 138$ Rdn. 4; vgl. allerdings zum „Terrainverlust“ der Privatautonomie im Gesellschaftsrecht zu Gunsten einer „fast lückenlosen gerichtlichen Zensur“, ReUTER, in: Bumke/Röthel, Autonomie im Recht, 2017, 118, 127; vgl. auch Bachmann, aaO (Fn. 75), S. 207, $222 \mathrm{f}$.

176 Zur Bedeutung des Zustandekommens des Vertragsschlusses für den Kontrollmaßstab vgl. etwa Grunewald, aaO (Fn. 23), S. 132.

177 Zum prozeduralen Grundcharakter der Vertragsgerechtigkeit und dem Verhältnis zu inhaltlichen Kriterien („Materialisierungstendenzen“) CANARIs, AcP (200) 2000, 273, $284 \mathrm{ff}$; vgl. auch WiEDEMANN, ZGR 1980, 147, 155 f; grundlegend auch LuHMANN, Legitimation durch Verfahren, 1969; Luhmann kontextualisierend Larenz, aaO (Fn. 140), S. 200 f.

178 So für Satzungsbestimmungen über Einziehung auch GEHrLeIN, NJW 2005, 1969, 1972.

179 So auch Grunewald, aaO (Fn. 23), S. 133; SchneIder, ZGR 1978, 1, 8.

$180 \mathrm{Zu}$ den Grenzen des Informationsmodells und des Selbstschutzes bei Eintritt in Kapitalgesellschaften, siehe umfassend Kuntz, aaO (Fn. 1), 3. Teil, B., $\mathbb{S} 1$, III, 3; Möslein, aaO (Fn. 74), S. $308 \mathrm{ff}$. 
fang der juristischen Beratung und wer - wenn auch wie oben gezeigt, das AGB-Recht keine Anwendung finden kann - der „Verwender“ der Vertragstexte ist. ${ }^{181}$ Abhängig vom Marktumfeld können Gründer allerdings gleichermaßen wie Investoren vertragsgestaltend sein; denn letztere wollen häufig auch die Kosten der Beteiligungskonzeption auf die übrigen Beteiligungsinteressenten abwälzen. ${ }^{182}$

Neben der Prüfung der Umstände des Zustandekommens des Rechtsgeschäfts bleibt die Ausübungskontrolle analog $\ 162$ Abs. 2 BGB ebenfalls relevant. ${ }^{183}$ Entscheidend ist auch bei einem Ausschluss auf Grund von Musterklauseln, ob bei deren Anwendung angemessen und transparent mit dem Gesellschafter umgegangen wird. Wird der Gesellschafter unvermittelt und über seinen Kopf hinweg ausgeschlossen, muss es das Unternehmenswohl unbedingt erfordern. Eine abgewogene und transparente Leistungsbewertung in Abstimmung mit dem betroffenen Gesellschafter ist hingegen Indiz für eine angemessene Ausübung. Dazu gehören, im Lichte des Gleichheitssatzes und der starken Betroffenheit bei Entzug der Gesellschafterstellung, Transparenz bei der Setzung von Mile-Stones im Business-Plan und im Feedback, die nachvollziehbare Begründung von Entscheidungen, Anhörungen und auch externe Schlichtungsversuche.

\section{Exemplarische Anwendung der modifizierten Hinauskündigungsdogmatik}

Die vorstehend entwickelten Maßstäbe sollen im Folgenden exemplarisch auf die beiden wesentlichen Typen von Vesting-Klauseln - Bad- und Good-Leaver-Fälle - angewendet werden.

\section{a) Bad-Leaver-Fälle}

Bad-Leaver-Fälle treffen den Gesellschafter am härtesten. Er verliert hier unabhängig von der Vesting-Periode alle Call-Option-Anteile gegen Erstattung lediglich des Nennwerts. Sofern er weitere Anteile hält, kann er verpflichtet sein, sie zum - gegebenenfalls eingeschränkten - Verkehrswert abzugeben. Kann der betroffene Gesellschafter nicht nachweisen, dass der Bad-Leaver-Fall vom Marktstandard abweicht, so muss er ihn inhaltlich gegen sich gelten lassen. Kann er allerdings stichhaltige Gegenproben zur Marktüblichkeit der Bad-Leaver-Klausel beibringen, etwa durch Vorlage von abweichenden Klau-

181 So Huber, ZGR 1980, 177, 210.

182 https://wwrw.vc-magazin.de/venture-capital/beteiligungsvertraege-im-venture-capital-sektor/ (letzter Zugriff: 4.1.2021).

183 Siehe schon oben V. 1. 
seln, die durch marktbekannte VC-Funds und Kanzleien verwendet werden, oder durch Sachverständigengutachten aus den genannten Kreisen, so ist in die inhaltliche Prüfung der Klausel einzusteigen. Hierbei gelten folgende Leitlinien.

Wirksamkeitswahrend wirkt sich aus, wenn der Tatbestand klar ist und einen sachlichen oder sogar wichtigen Grund enthält. Um eine Nähe zu Missbrauch oder unangemessener Freiheitseinschränkung zu vermeiden, muss die materielle Tatbestandsverwirklichung weitestgehend in den Händen des Gesellschafters liegen. Die Kündigung des Anstellungsverhältnisses aus wichtigem Grund, der hinreichend durch die Rechtsprechung präzisiert ist und auch ein Verschuldensmoment enthalten kann, ist zulässiger Bezugspunkt. Problematischer tatbestandlicher Anknüpfungspunkt wäre dagegen eine ordentliche Kündigung seitens der Gesellschaft und auch die Eigenkündigung. Letztere wurde im Zusammenhang mit den „Management-Modellen“ unter dem Gesichtspunkt einer unzulässigen Kündigungsbeschränkung nach $\ 626$ Abs. 4 BGB kontrovers diskutiert. ${ }^{184} \mathrm{Ob}$ die Eigenkündigung wegen Krankheit von Nahbezugspersonen des Gesellschafters, Burn-Out oder reinem wirtschaftlichen Opportunismus eintritt, wäre bei einer starr auf den Rechtsakt der Kündigung abstellenden Klausel nicht berücksichtigt. Auch die bloße Beendigung der Tätigkeit ist nur schwer als Tatbestand eines Bad-Leaver-Falles haltbar, da diese durch Freistellung oder Abberufung einseitig durch die Gesellschaft bewirkt werden kann. ${ }^{185}$

Bei ausdifferenzierten Tatbeständen hingegen, etwa bei der Verfehlung von Milestones im - wie auch immer ambitionierten - Businessplan, dürfte neben einem Verschuldensanteil, umso mehr entscheidend sein, wie die Klausel vereinbart wurde, etwa ob der betroffene Gesellschafter fachkundig beraten war, er im Verbund mit weiteren "Gründern" das Vertragsmuster eingebracht hat oder erst später zur Gesellschaft gestoßen ist und keine reale Möglichkeit hatte, die bestehende Nebenabrede zu verändern. Entscheidend dürfte in Fällen, in denen der Gesellschafter den Tatbestand kontrolliert, sein, dass er sich bewusst in das Gesellschaftsverhältnis begibt. Der Verlust von Call-Options-Anteilen zum Nennwert realisiert dann ein kalkulierbares Risiko. Die Anteile wären nur als erdiente Vergütung im Vermögen des Gesellschafters verblieben. Das Abfindungsgebot stellt die Wirksamkeit des Vesting-Regimes nicht in Frage, weil die Chance auf den Verbleib der Anteile als solche Vergütungscharakter hat. Es geht nicht um den Fall der Entziehung bestehender Anteile, sondern, in der Logik des „umgekehrten Anwartschaftsrechts“, um die Versagung des endgültigen Erwerbs. Die Abfindungsansprüche zu Verkehrswert können sich

184 Vgl. umfassend dazu Habersack/Verse, ZGR 2005, 451, $466 \mathrm{ff}$; siehe auch das Beispiel in Samios/Arnold, aaO (Fn. 23), S. 148.

185 Vgl. Stenzel, aaO (Fn. 23), Rdn. 612. 
daher wirksam auf die erdienten und vor Beginn des Vesting bereits gehaltenen Anteile beschränken. ${ }^{186}$ Denn Abfindungszahlungen zum Verkehrswert können den Bestand des Unternehmens gefährden. Im Übrigen wäre der Verkehrswert der Anteile im Startup-Umfeld auch nur sehr schwer zu ermitteln, gegebenenfalls freilich durch Rückgriff auf die in einer Finanzierungsrunde besprochenen Werte, wenngleich sie zum Ausschlusszeitpunkt häufig weit in der Vergangenheit liegen und daher ungenau sein können.

\section{b) Good-Leaver-Fälle}

Good-Leaver-Klauseln regeln den Verlust noch nicht erdienter Geschäftsanteile. Insbesondere wenn die Cliff-Periode noch nicht erreicht wurde oder auch der Status der Founder-Anteile verändert wird, können auch sie die Stellung des Gesellschafters schwer beeinträchtigen. Das Zeitmoment der CliffPerioden ist ein objektiver Faktor, so dass ein Abgleich mit dem marktüblichen Standard verhältnismäßig einfach möglich ist. Hier ist von einer üblichen Dauer bis höchstens einem Jahr auszugehen, in dem jeder Leaver-Fall zum vollständigen Anteilsverlust führt. ${ }^{187}$

Der Schutz der Investoren vor Ansprüchen nur kurzfristig im Unternehmen engagierter Gesellschafter und Mitarbeiter ist legitim und entspricht dem Leistungsprinzip. ${ }^{188}$ Eine genaue Ausübungskontrolle ist allerdings vorzunehmen, wenn eine Call-Option kurz vor Ablauf der Cliff-Periode ausgeübt wird; danach liegt der Bewertungsschwerpunkt wieder auf dem Tatbestand selbst. Good-Leaver-Fälle betreffen die Substanz, indes nicht den Kern der Gesellschafterstellung. Häufig geregelt sind Tod, Insolvenz und Eigenkündigung oder Kündigung ohne wichtigen Grund. Stellt eine Leaver-Klausel auf die Kündigung des Dienstverhältnisses ab, kann das ordentliche Kündigungsregime des Arbeitsverhältnisses den sachlichen Grund der Klausel sicherstellen. Denn sofern das Kündigungsschutzgesetz greift, muss die Weiterbeschäftigung objektiv unzumutbar sein. ${ }^{189}$ Bei kleineren Unternehmen ist das allerdings häufig nicht der Fall, so dass eine voraussetzungslose Kündigung nach $\ 620$ Abs. 2 BGB möglich ist. Ein analoges Problem besteht bei Anknüpfung der Leaver-Klausel an die Abberufung als Geschäftsführer, da diese voraussetzungslos ist, sofern keine Beschränkung nach $₫ 38 \mathrm{GmbHG}$ vorliegt. In diesen Fällen fehlt es an einer Kontrollierbarkeit des Tatbestandes für den

$186 \mathrm{Zu}$ wenig differenzierend etwa Weitnauer/Grob, GWR 2015, 353, 355.

187 So auch Bank/Möllmann, aaO (Fn. 23), Teil 3, Art. 5 Rdn. 266.

188 Dazu etwa Habersack/Verse, ZGR 2005, 451, $468 \mathrm{ff}$.

189 BGH NJW 2005, 3644, 3645f; Hergenröder, Münchener Komm. z. BGB, 8. Aufl., 2020, \1 KSchG Rdn. 75; vgl. aber zum Kündigungsschutz für Geschäftsführer BoEMKE, RdA 2018, 1, $17 \mathrm{ff}$. 
Gesellschafter. Hier muss besonders berücksichtigt werden, ob die Ausübung der Call-Option vor oder nach Ablauf der Cliff-Periode erfolgt, wie die Klausel zustande gekommen ist, insbesondere ob ein Vertragsmuster vom Gesellschafter selbst eingebracht wurde und wie das Investitionsrisiko wegen eigener Vorleistungen des betreffenden Gesellschafters reduziert ist.

\section{Fazit}

Die Unternehmensfinanzierung durch Venture Capital hat weitreichende Implikationen. Die ökonomischen Leitmotive „Investition gegen Gesellschaftsbeteiligung" und "Arbeit gegen Gesellschaftsbeteiligung" schlagen sich exemplarisch beim Vesting nieder und führen zu einem Funktionswandel von Geschäftsanteilen hin zu einem Marktinstrument. Die Gesellschafter finden sich in einer besonderen Anreiz- und Disziplinierungsstruktur wieder, die von formularmäßigen Verträgen und einer verbreiteten Übung bestimmt wird. Dies muss bei der Gestaltung von Beteiligungen sowie ihrer rechtlichen Bewertung berücksichtigt werden, insbesondere um eine weitere Schwächung staatlichen Gerichtswesens gegenüber der privaten Schiedsgerichtsbarkeit zu vermeiden. Zudem spielt die Anerkennung der VC-Beteiligungspraxis im internationalen Wettbewerb um hochqualifiziertes Personal und Investoren eine wichtige Rolle für deutsche GmbHs. Der Rechtsstandort Deutschland sollte sich nicht der internationalen Dynamik verschließen, wobei die privilegierten Besteuerung von Mitarbeiterbeteiligungen unter dem Fondsstandortgesetz ein erster Schritt in die richtige Richtung ist. ${ }^{190}$ Die hier aufgestellten Thesen können auf materiell-rechtlicher Ebene zur Absicherung der im Markt verbreiteten Investitionslogik beitragen und damit das deutsche Gesellschaftsrecht im Wettbewerb der Rechtsformen stärken. Ob sich die hiesigen Ergebnisse auf andere Bereiche des Wirtschaftslebens übertragen lassen, kann hier nicht vertieft werden, scheint jedoch naheliegend. Hier sind zunächst folgende Thesen festzuhalten:

1. Auf Vesting-Regime kann die Dogmatik zur Hinauskündigung von Gesellschaftern angewendet werden. Sie ist allerdings wegen der besonderen Motivlage bei Wagnisbeteiligungen als Investitions- und Vergütungsordnung zwischen gleichgeordneten Parteien um eine wirtschaftssoziologische Betrachtungsweise zu modifizieren.

2. Da Wagnisbeteiligungen häufig auf branchenüblichen Mustern beruhen und insoweit eine Handelsübung transportieren, ist ein „judicial self-restraint“ bei der Inhaltskontrolle angezeigt. Handelsbräuche sind wegen ihrer normativen Begründung nicht sittenwidrig. Die Prüfungsintensität ist daher zurückzuneh- 
men, wenn Ausschlussklauseln auf üblichen Mustern beruhen. Für in solchen Mustern kondensierte Handelsbräuche sollte eine Wirksamkeitsvermutung gelten. Ausschlussstreitigkeiten verlagern sich daher vom Nachweis eines sachlichen Ausschlussgrundes zum Nachweis der Marktüblichkeit der Ausschlussklausel.

3. Die Inhaltskontrolle lebt wieder auf, wenn dargelegt ist, dass eine Klausel nicht dem Marktstandard entspricht. Die Umstandskontrolle bezüglich des Zustandekommens einer Klausel und ihrer Ausübung bleibt hingegen auch bei marktüblichen Klauseln stets offen.

4. Im Interesse der Rechtssicherheit sind diejenigen Regelungsthemen bei Wagnisbeteiligungen zu typisieren, bei denen eine Abweichung von der Marktpraxis besonders schwerwiegend ist. Hier wurden in einem ersten Ansatz die Reichweite des Anteilsverlusts und die Kontrolle der Leaver-Tatbestandsverwirklichung als solche identifiziert.

5. Die Übertragung der vorgenannten Leitlinien auf andere Instrumente der VC-Finanzierung liegt nahe, wenn auch sie auf in Musterklauseln geronnenen Handelsbräuchen beruhen. 